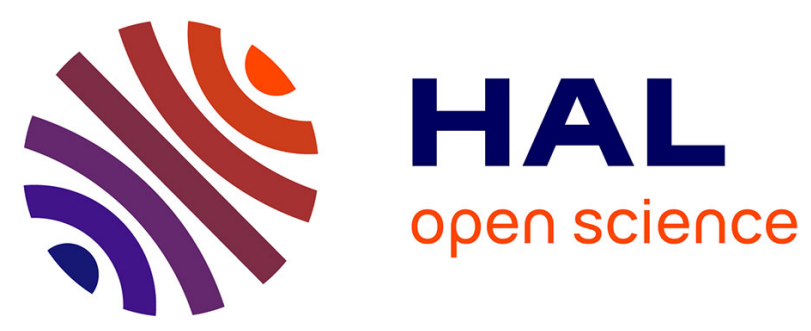

\title{
Graphical symbol recognition
}

\author{
Santosh K.C., Laurent Wendling
}

\section{To cite this version:}

Santosh K.C., Laurent Wendling. Graphical symbol recognition. Wiley, pp.1 - 22, 2015, Encyclopedia of Electrical and Electronics Engineering, 10.1002/047134608X.W8260 . hal-01166512

\section{HAL Id: hal-01166512 \\ https://hal.science/hal-01166512}

Submitted on 23 Jun 2015

HAL is a multi-disciplinary open access archive for the deposit and dissemination of scientific research documents, whether they are published or not. The documents may come from teaching and research institutions in France or abroad, or from public or private research centers.
L'archive ouverte pluridisciplinaire HAL, est destinée au dépôt et à la diffusion de documents scientifiques de niveau recherche, publiés ou non, émanant des établissements d'enseignement et de recherche français ou étrangers, des laboratoires publics ou privés. 


\title{
Graphical symbol recognition+
}

\author{
K.C. SANTOSH ${ }^{\ddagger}$
}

National Library of Medicine, $\mathrm{NIH}$

8600 Rockville Pike, Bethesda, MD 20894, USA

LAURENT WENDLING $\ddagger$

SIP-LIPADE, Université Paris Descartes (Paris V)

45, rue des Saints-Pres, 75270 Paris Cedex 06, FRANCE

Email: santosh.kc@nih.gov \& laurent.wendling@parisdecartes.fr

‡Corresponding authors (both can be contacted)

${ }^{\dagger}$ Citation. Santosh, K.C. and Wendling, L. Graphical Symbol Recognition. Wiley Encyclopedia of Electrical and Electronics Engineering. p. 1 -22, 2015, DOI:10.1002/047134608X.W8260

Abstract. The chapter focuses on one of the key issues in document image processing i.e., graphical symbol recognition. Graphical symbol recognition is a sub-field of a larger research domain: pattern recognition. The chapter covers several approaches (i.e., statistical, structural and syntactic) and specially designed symbol recognition techniques inspired by real-world industrial problems. It, in general, contains research problems, state-of-the-art methods that convey basic steps as well as prominent concepts or techniques and research standpoints/directions that are associated with graphical symbol recognition.

Keywords. Graphics processing · graphical symbol · recognition, retrieval and spotting · statistical approaches · structural and syntactic approaches $\cdot$ real-world problems.

\section{Introduction}

Document analysis or processing is mainly related to texts and graphics. It concerns text and or graphics separation, localization and recognition (1). According to (2), document analysis is related to document image analysis (DIA) since both research works have been concerned with document image interpretation. In a similar manner, (3) categorize document image analysis into two domains:

1) textual processing and

2) graphics processing. 
In both articles $(2 ; 3)$, the basic tasks are image segmentation, layout understanding and graphics recognition. Graphics recognition has been the subject of intensive research activity in the community of pattern recognition and document analysis. Graphical symbol recognition, in particular, has a long history since the 70's, and it is considered as the core part of graphical document image analysis and recognition systems. In 1998, $(4 ; 5)$ a prominent researcher has made a statement: 'none of these methods works' in general. Since then, it has been actively extended (5-9). Very recently, the importance and the usage of graphics recognition have been reported (9).

Graphics or graphical symbols are referred to as visual images or designs, interpreting information about the context. Graphical symbols are generally 2D-graphical shapes, including their composition in the highest level of conceptual information. Overall, it plays a crucial role in a variety of applications such as automatic interpretation and recognition of circuit diagrams (10; 11), engineering drawings and architectural drawings (12-15), line drawings (16), musical notations (17), maps (18), mathematical expressions (19), and optical characters (20-23). Graphics is often combined with text, illustration, and color. Therefore, in document image processing, graphical symbols, for instance, convey crucial cues about the context in comparison to texts. Besides generic approaches, text recognition is distinct from symbol recognition, even though their boundaries are not obvious. Thus, their solutions complement each other $(1 ; 24)$.

In general, the whole symbol recognition process is based on either

1) matching features between a query and dataset symbols or

2) comparing decomposed parts such as lines and arcs as well as the relations between them.

These are commonly referred to as statistical and structural approaches, respectively. An overview of the performance of the most commonly used shape descriptors (refer to the statistical approaches) for symbol representation is provided in (25). In case of structural approaches, to recognize graphical symbols and/or to localize significant or known visual parts of the technical documents or images, graphics processing refers to low level primitives analysis. On the other hand, it is concerned with regions-of-interest analysis and recognition. This means that research scientists take a graphical symbol as a set of primitives, which may be lines or arcs, triangles, rectangles, or more complex primitives like loops $(14 ; 26 ; 27)$. Their interpretations, however, depend on the studied application as well as on their specific local context. Therefore, primitive extraction and symbol recognition, on the whole, are the key steps toward understanding and interpreting content within a document. Considering both approaches, researchers addressed the use of their best possible efficient combinations $(27 ; 28)$, which has been recently reported (8):

'... the recurring wish for methods capable of efficiently combining structural and statistical methods' and 'the very structural and spatial nature of the information we work with makes structural methods quite natural in the community.'

The problem can further be extended to symbol spotting, but one can view this as a kind of retrieval (2933), which is guided by user queries. Additionally, the recognition/retrieval process can be made with the help of local descriptors like scale invariant feature transform (SIFT) and with the use of techniques like bag-of-features so that either primitive or region extraction (segmentation) can be avoided. The question always remains the same, 'what approach does what (i.e., performance) in which context?'.

As mentioned earlier, graphics recognition has an extremely rich state-of-the-art literature in symbol recognition and localization $(6 ; 9 ; 34 ; 35)$. But the major state-of-the-art methods for symbol recognition, 
do not conclude on the existence of a set of generic methods that can yield the best results, even though they are easy to implement (with fewer parameters) and are reproducible. This brings an idea of complexity variations between the samples from one application to another. Another reason could be the industrial project restriction, which requires automated systems with higher accuracy to reduce the cost of human intervention and ensure the effectiveness of the product. As a consequence, generic approaches might be adjusted to process the type of application under consideration. Since industrial partners are focusing on very specific problems, there exists several contracts and collaborations for research scientists. For example, industrial projects are related to information retrieval or document reverse engineering, associated with more powerful computers and the development of enormous storage capacity, providing huge attention from the scientific community for recognizing symbols in technical documents (35-38). The latter context denotes graphics recognition, an active research field, requires constant advances so that scalability problems, for instance, can be handled. This also explains why the best approaches found in the literature refer to a specific application context and are guided by a priori knowledge of the documents processed.

In Section 2.1, we provide a detailed review of common steps that are employed in the state-of-the-art methods for graphical symbol recognition. In Section 2.2, we highlight major projects that allows to forecast real-world industrial scenarios. State-of-the-art methods are enumerated in Section 2.3.

\section{Graphical symbol recognition system and applications}

\subsection{Basic steps in system design}

The recognition of graphical symbols or any meaningful shapes has been the subject of numerous reviewed research articles $(13 ; 24 ; 39-43)$. Most of these proposed systems are roughly described using the following two major units: 1) data acquisition and preprocessing; and 2) data representation and recognition.

\subsubsection{Data acquisition and preprocessing}

The documents are usually scanned at grayscale with high possibility of noise, distortion and deformation. When considering raster data, acquisition and preprocessing tools are aimed at reducing the amount of data to the most 'relevant' information to efficiently locate the regions-of-interest in an image. A binarization step $((44 ; 45)$, for example) is then performed to extract useful information from the background. This is important since it influences the quality of subsequent processing algorithms that are dedicated to data representation and recognition. Similarly, foreground and background separation can be taken as another important tool (46). Without these, defects such as creases, stains on paper and heterogeneous wear, for example, can lead to inconsistent results. Therefore, they must be corrected with specific operators as a precursor or incorporated into the appropriate data acquisition and preprocessing unit. For correction, it may require human expertise and automatic spectrum of possible damages and their quantification. The definition of criteria and their evaluation for the quality interpretation of documents is also a challenging issue. This means that the data from this unit may undergo further processing consistent with the application under consideration. For example, the application can be either text and graphics separation (47-53) or simply pruning by removing small connected components. In other words, to focus on graphics efficiently, one needs to separate texts from the document images. Text/graphics separation aims at segmenting document into two layers so that one can focus on interest regions directly, where graphical symbols lie. The usefulness of text/graphics separation can be found in (54). Several methods were proposed to extract text parts from background to segment documents (9). Among them, the method proposed by Fletcher and Kasturi (1), has been widely used as it provides promising results in most cases, including wide ranges of applications. The 
main problem of their method, however, relies on a priori knowledge about special characters and the size of connected components, since it may omit to put little disconnected symbols (or graphical shapes/elements) in the graphical layer. In (52), authors proposed to advance earlier performance by considering local segmentation. Recent promising advancements consider sparse representation to tackle this problem (55).

To achieve the optimal performance from the subsequent processing algorithms, this unit requires the most $a$ priori knowledge about the document structure and the symbol representation.

\subsubsection{Data representation and recognition}

In general, data representation and recognition steps are strongly correlated with the preprocessing step. The quality preprocessor yields quality data at the output so that one can efficiently represent it for recognition.

As mentioned in Section 1, the graphical symbols are represented either in the form of feature vectors by estimating the overall shape or in more structured forms (i.e., graphs) by using meaningful primitives that are extracted from the whole symbol. In the latter case, primitive selection tools are application dependent. Data quality and the problem complexity determine the data representation, which is again followed by matching techniques to be used in the decision process. A good data representation is assumed to be compact and discriminant, and minimizes the intra-class distance and maximizes the inter-class distance (6). Further, feature computation must have realistic time complexity as well as storage format to ensure data portability. This ultimately makes classification easier. Classification is the problem of identifying to which of a set of classes a new query belongs. This is basically based on a training set of data containing samples whose class membership is known.

Existing approaches, specifically those based on feature based matching, can be split into three categories: statistical, structural and syntactic. Most methods are particularly suited for isolated line symbols, not for composite symbols connected to a complex environment $(6 ; 13 ; 35 ; 56)$. As said before, these are guided by how the symbols are represented. Further, in each category, we have found that the choice of methods often depends on human expertise. To support this statement, we summarize the reasons in the following with examples.

1) The techniques used in statistical approaches fairly computes the differences between two feature vectors $(56 ; 57)$.

2) The techniques based on graph grammar will be more suitable to search symbols in technical documents where information is close to a feature vector description that follows composition rules of primitives (58-64). On the other hand, the graph matching techniques are also effective to handle vector-based representation of primitives (attributed relational graphs (ARG), for example) $(13 ; 65)$.

On the whole, following the state-of-the-art methods, we have neither seen any absolute standard on the choice of the best approach, nor detected obvious combinations of techniques for optimal performance. A detailed study is provided in Section 2.3.

\subsection{Applications: real-world examples}

Over the decades, international association of pattern recognition (IAPR) sponsored graphics recognition (GREC) workshops, supported by technical committee 10 (TC-10: http: / / iapr-tc10 . univ-lr.fr/) have been organized together with the contests, where graphical symbol recognition, retrieval and spotting are the prominent tasks. Besides regular papers in the proceedings, these 

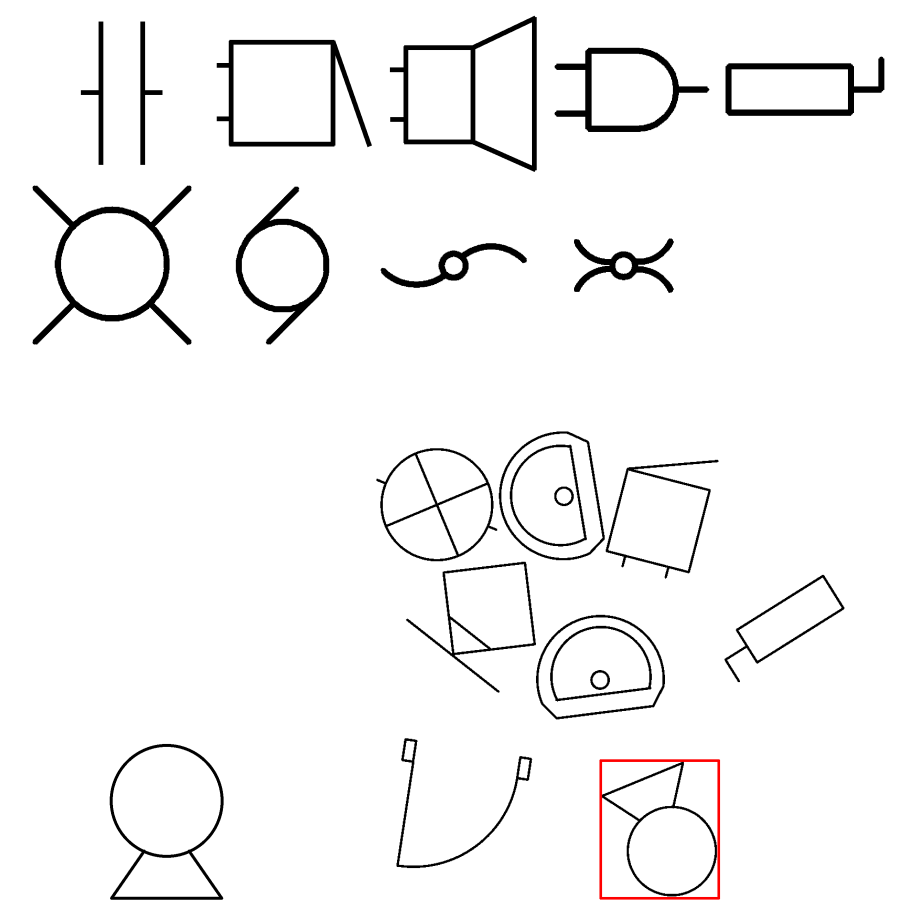

(a) Query symbol (b) Symbol recognition and retrieval

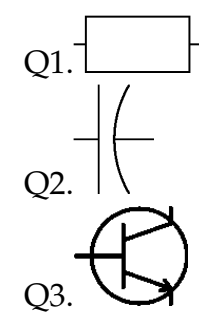

(a) Query symbols

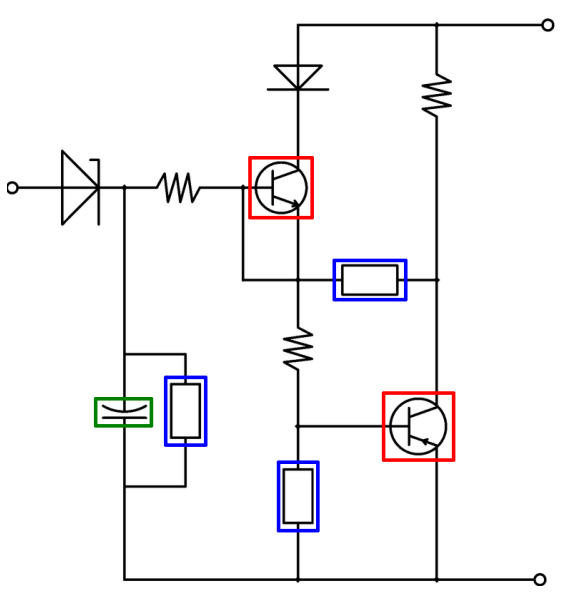

(b) Symbol spotting
Fig. 1. Graphical symbols. An example illustrating lineal and fully isolated symbols (66).

Fig. 2. Isolated graphical symbols in the form of bag (31). In this illustration, the task is to recognize (exactly) similar symbol(s) in response to the applied query.

Fig. 3. Graphical symbols, appearing in the circuit diagram (31). The aim of the task is to spot the symbol(s) that is(are) similar to the applied queries. In this example, expected symbol spotting outputs are shown by using bounding boxes in three different colors: one color per query.

GREC contests aim to objectively evaluate the state-of-the-art of graphics recognition techniques and to generate performance evaluation tools and datasets for future research $(31 ; 67-69)$. Fig. 1 shows a few model symbols $(66 ; 70)$. The dataset also contains other categories such as rotated, scaled, deformed and degraded at different levels, which are then used to recognize by matching with the model symbols. In general, considering isolate symbols, the aim of the contest is to realize how well the symbols are represented: are the representations invariant to rotation and scaling, and robust to deformed and degraded symbols?

Unlike the GREC contests, symbol recognition problems often rely on industrial context. Considering a real-world problem, symbol recognition is not straightforward. Straightforward symbol recognition means that any test symbol is matched with model symbols, and the model symbol with the highest similarity score is selected. Techniques that are used to automate the systems, however, are guided in accordance with the need of the collaborators. In general, common applications are recognition and 
Fig. 4. Graphical symbols, appearing in the floor plans (31). An example showing symbol spotting problem in floor plan.

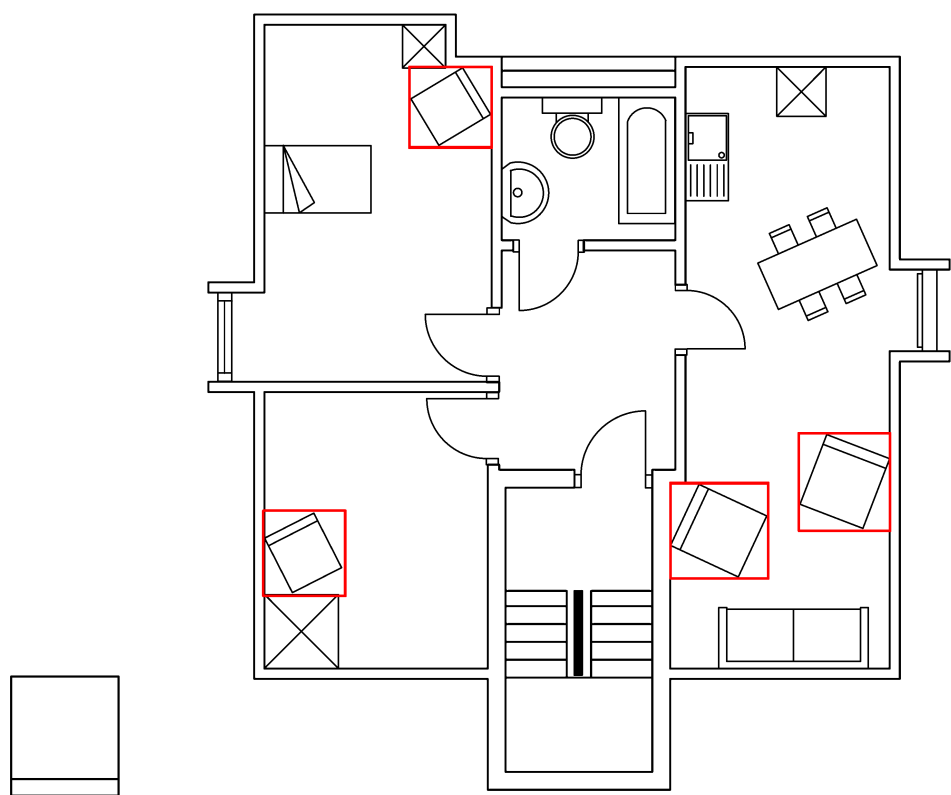

(a) Query symbol

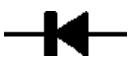

(a) Query symbol
T1. T2 T3.

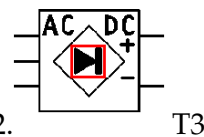

(b) Graphical symbol/element spotting in test images (T1:T4)

T4.
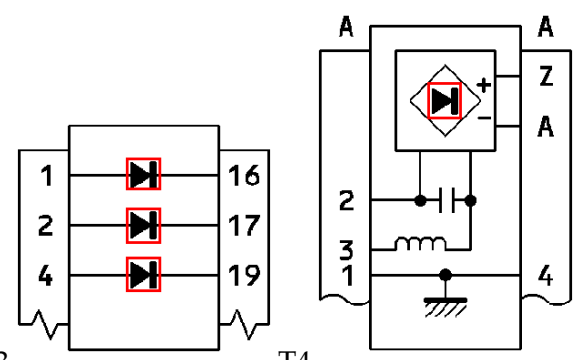

(b) Symbol spotting

Fig. 5. Graphical symbols. An example illustrating the complexity of the FRESH dataset $(27 ; 71)$, starting with an isolated graphical symbol (moving from left to right). This example shows how the known and significant part can be spotted based on the applied query.

localization of graphical symbols in electronic documents, in architectural floor plans, wiring diagrams and network drawings (see Figs. 2, 3, 4 and 5) $(6 ; 27 ; 31)$. In Fig. 2, one can see the problem, quite different from GREC 2003 and 2005 contests - as test symbols can be queried to recognize similar ones from the bag of symbols. In a single bag, symbols can be repeated and therefore, this can be taken as a retrieval problem. In Fig. 3, an example of symbol spotting is shown. Like in Fig. 2, a query symbol is applied to detect similar symbols from a complete circuit diagram. A similar problem is provided in Fig. 4, where the task is used to detect symbols which are similar to the query. This example shows a architectural floor plan, a completely different application. In Fig. 5, the task characterizes differently in comparison to the earlier ones even though the aim remains the same. These samples (entitled as FRESH dataset) are taken from (71). The symbols may either be very similar in shape - and only differ by slight details - or be completely different from a visual point of view. Symbols may also be composed of other known and significant symbols and need not necessarily be connected. For such complex and composite symbols, as before, an isolated query symbol is applied not only to recognize similar symbols from the dataset, but also to detect known and significant parts (graphical elements) which are associated with the query symbol. In the state-of-the-art, the latter issue has been considered as the most challenging problem. Applications can also be extended to extract very specific graphical elements: arrows (i.e., pointers, in general), and dotted lines to highlight regions-of-interest (16; 72-74). 

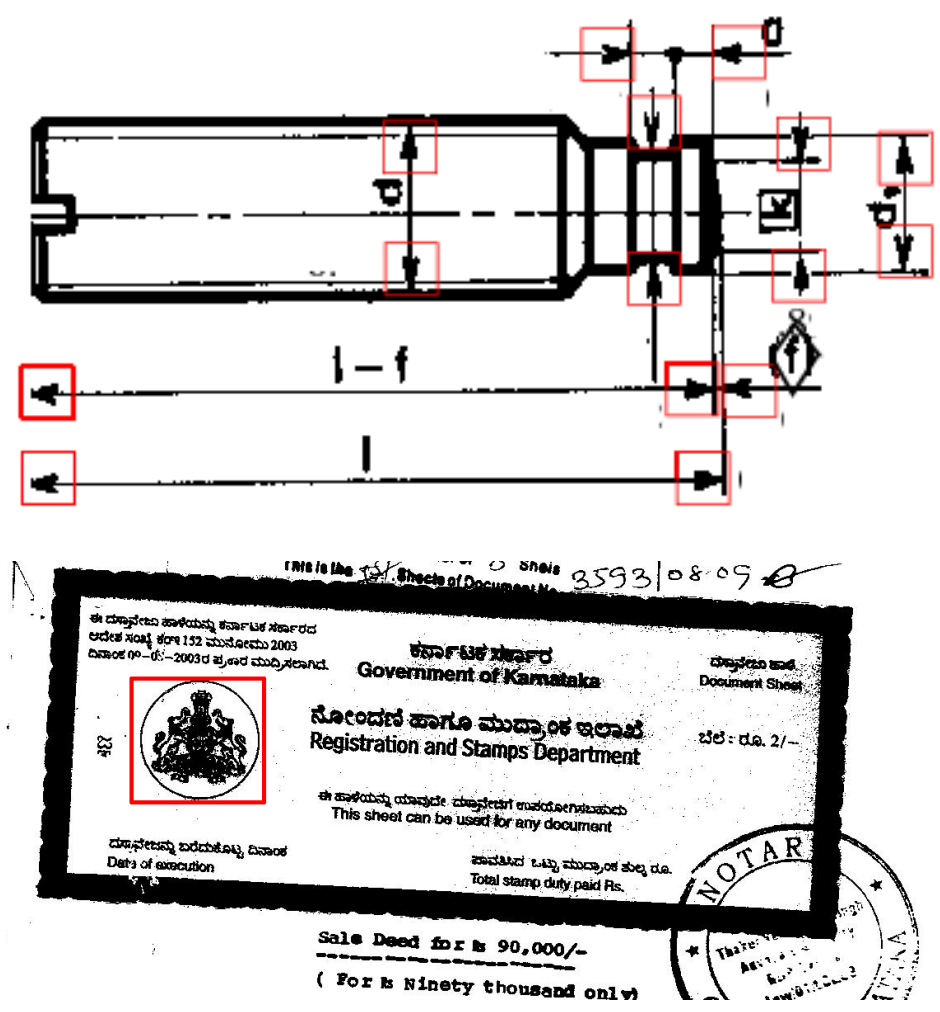

Fig. 6. Arrow detection (using a cropped document image), another important task in graphics recognition (16). Arrow detection locates important quotation and of course, meaning parts.
Fig. 7. Logo detection, a precursor to document indexing, classification and content-based image retrieval. The region that contains logo is cropped from a document image, for a better look.

Fig. 6 shows an example of it.

Like other applications, logos, stamps and/or seals are important graphical features, which are used to index, classify documents within the framework of content-based image retrieval (CBIR) problem (75). This means that searching administrative documents based on any particular logo/seal (as a query) allow quick filtering or routing of the document to the right business process, archive or individual (76-80). These methods are promising in detecting objects in general in terms of accuracy, time and scalability since symbols, such as seals and logos are synthetic entities consisting of uniform regions and these are highly structured. As a consequence, logo/seal detection advances the performance of document retrieval $(81 ; 82)$. An example of logo detection is shown in Figure 7.

Based on these, we refer to this issue as a generic problem recognition, in either the parts or the whole graphical symbols $(6 ; 27 ; 31 ; 32 ; 83 ; 84)$. The aforementioned applications require strong knowledge about how we can represent graphical symbols and recognize, detect or spot them.

\subsection{Symbol representation and recognition}

Automatic recognition, description, classification and grouping of patterns are important issues in many disciplines such as biology, computer vision, artificial intelligence or remote sensing where pattern representation (shape analysis, for example) plays an important role $(42 ; 85)$. Patterns can be graphical elements like symbols (56) or other visual structures of any kind including cursive characters (86). Broadly, to represent any pattern, any one or the combination of the following three different approaches is used in the state-of-the-art:
1) statistical,
2) structural and
3) syntactic approaches. 
In general, the choice of the approach relies on the complexity of the problem. In Sections 3 and 4 , we explain in detail the aforementioned three approaches.

\section{Statistical approaches to symbol recognition}

\subsection{Common methods}

In statistical approaches, shape analysis plays a crucial role in graphical symbol recognition (35; 56). Shape features are often grouped into two families (85):

1) contour-based descriptors and

2) region-based descriptors.

There are several approaches to describe boundary contours based on a small set of features, and the choice is driven by requirements such as robustness to noise and tolerance to small distortions. In contour-based descriptors, one of the most commonly used techniques is the Fourier descriptor (FD) (87-92). These descriptors can be easily integrated due to their simplicity and robustness in many applications dedicated to character recognition (93). Others are based on polygonal primitives from contour information and have been used for matching via dynamic programming (94; 95). A polygonal approximation of any shape results in a loss of information (for instance, the degree of ellipticity is not well suited for recognition of polygons (94)). In (96), another approach has been presented based on a contour-oriented $2 D$ object where recognition shows robustness towards polygonal approximation inconsistency. The major drawback however, is its high time complexity. The generalized Hough transform (97-99) can be taken as a useful technique but high computational time and storage requirements are its substantial issues. From a practical point of view, the work presented in (99) does not execute faster. But in (100), both time and space complexity are reduced by targeting the regions-of-interest. Curvature approaches (101-104) describe shapes in scale space. In $(101 ; 102)$, a shape is described in a scale space by the maximum of the curvature feature vector, considering the object's boundary contours. The similarity between shapes can be measured by the distance between their corresponding scale space representations. For instance, shape context (SC) (105) is robust to small perturbations while it does not guarantee scale-invariance. Skeleton extraction has also been used for pattern matching $(106 ; 107)$ where shapes can be defined as a variant of contour-based approaches. In these approaches, as reported in (107), each instance of an object is represented by a graph built on the medial axis of the shape silhouette. Improved versions of the graph have been proposed (108-110) with the shock graphs, and several works have focused on methods to efficiently match two graphs $(101 ; 107)$. These methods are highly effective since they are based on global optimizations. However, they have a high complexity and comparative studies show that they are less tolerant to scale variation than polar curvature methods (104). For a more detailed discussion of graph matching, we refer to Section 4 . In short, all these contour-based descriptors are

1) appropriate for silhouette shapes, and

2) limited to certain applications since they cannot capture interior contents, disconnected shapes or shapes with multiple holes where boundary information is not available.

Region-based descriptors take into account all pattern pixels. Common methods are based on the moment theory including geometric, Legendre, Zernike, and pseudo-Zernike moments (111-119). Invariant moments were introduced in 1960 (120). Comparative studies $(111 ; 114)$ have demonstrated 
interest in improving invariance properties (rotation, translation and scaling) $(112 ; 119 ; 121-123)$ and reducing execution time of the Zernike moments (124). Before that, in (117), the author focused on orthogonal moments, which are computed from either binary or grayscale image. This property facilitates to reconstruct the studied image (118). Zhang and $\mathrm{Lu}$ (125) have proposed a region-based generic Fourier descriptor (GFD) to overcome the limitations of contour-based FD. Since this approach uses the 2D Fourier transform (FT) on a polar-raster sampled shape, it can also avoid the problem of rotation in the Fourier spectra. Experimental results show that GFD outperforms common contour-based (classical Fourier and curvature approaches) and region-based (Zernike moments) shape descriptors (125). The main drawback of these approaches is their high processing time despite the possibility of optimization and complexity reduction $(124 ; 126)$. On the whole, region-based descriptors can be summarized as follows:

1) A normalization procedure (representing global shape by a single vector, for instance), in region-based descriptors is used to satisfy common geometric invariance properties by using centroid position, re-sampling and re-quantization, for instance.

2) Such a single feature vector, on the other hand, may not always capture all shape information, and eventually becomes less discriminant. However, discrimination power and robustness strongly depend on the selection of an optimal set of features as well as proper fusion of classifiers (127-130). For instance, in the field of symbol recognition, to increase performance, descriptors have been combined with several classifiers according to (131).

On the whole, it is not trivial to make a strict categorization of state-of-the-art into contour- and region-based methods since their mathematical/theoretical background can be similar to each other. But the choice depends on the need and the complexity of the studied problem.

\subsection{Symbol recognition related methods}

In statistical approaches, two aspects are primarily involved: feature selection and model selection for recognition. Usually each pattern is represented as an $n$-dimensional feature vector i.e. $\mathbf{x}=$ $\left(x_{1}, x_{2}, \ldots, x_{n}\right) \in \mathbb{R}^{n}$ and recognition is carried out by partitioning the feature space into different known classes.

In symbol recognition, geometric features such as centroids, axes of inertia, circularity, area, line intersections, holes and projection profiles, geometric moments and image transformations are used to recognize different patterns such as graphical symbols and characters. Within this framework, shape descriptors take into account the global appearance of a pattern. A comprehensive explanation can be found in (132). However, the stand-alone geometric features such as the degree of ellipticity (corresponding to the ratio of major to minor axis) (117), the maximum orientation axis (133) and fuzzy topological attributes (134-136) may not be suitable for covering broad graphics recognition problem. But they are still useful for separating symbols having distinct shapes (56). The angular radial transform (ART) (137), a part of MPEG7 - a powerful method (computed by projecting image on an orthogonal basis) - allows the user to obtain optimal performance for graphical symbol recognition (138).

Global shape representation is widely used because of its implementation simplicity since it does not require extra pre-processing and segmentation, in contrast to local pattern representation (85). In (37), global shape descriptors are studied for symbol recognition to reduce processing time, where the reported results (using multi-oriented and multi-scale character dataset) are generally better than Zernike moments and circular features (139). The Radon transform (RT) has also been widely used to globally describe an object by using all possible projections (140), which is a particular case of the 
Trace transform (141) to represent patterns. In this application, the RT is combined with the distance transform to take the description of angular objects into account or by integrating the distance between the parts of objects in the regular scans of the space in a spectrum management (142-144).

In (144), the feature vector representing shape distribution of the studied pattern are computed after normalizing all possible projection profiles in the range: $(0, \pi[$. As a consequence, it provides fast matching at the cost of not offering complete shape information. Motivated by this, the histogram of the RT has been used instead of compressing them (i.e., profiles) into a single vector (145), assuming that the studied patterns are equal in size. We remind the readers that the Radon transforms are essentially a set of parametrized histograms. In contrast, in $(146 ; 147)$, authors used dynamic time warping (DTW) to align every histogram for each projecting angle to absorb varying histogram sizes resulting from image signal variations. Thanks to DTW, it avoids compressing the feature matrix into a single vector which would otherwise miss information. The latter method provides better results in comparison to the results reported earlier. DTW is a commonly used approach (148).

As said before, graphical symbol recognition requires the use of robust descriptors to obtain rich information. Developing a good descriptor is still an open issue. This is because of the high variability of appearance such as rotation, partial occlusions, elastic deformations, intra-class and inter-class variations, or high variability among symbols due to different writing styles. In (149), the authors introduce a shape descriptor, which is able to deal with the changes in appearance from which these types of symbols suffer. The shape of the symbol is aligned based on principal components to make the recognition invariant to rotation and reflection, in addition to the use of the blurred shape model descriptor (BSM), where new features encode the probability of appearance of each pixel that outlines the shape of the symbol. Later, the algorithm has been extended for mutliclass symbol recognition. In (150), authors describe another framework to learn a model of shape variability in a set of patterns. The framework is based on the active appearance model (AAM) and permits to combine shape deformations with appearance variability. They have combined two modifications of the BSM descriptor as basic shape and appearance features to learn the model. These modifications allow to overcome the rigidity of the original BSM, and adapt it to the shape deformations. They reported that the results of the proposed methodology outperform the original BSM approach.

In (151), authors proposed a new descriptor by constructing a histogram for every pixel to figure out the distribution of constraints among the other pixels. These histograms are then statistically integrated to form a feature vector. This descriptor is well suited for symbol recognition in technical drawings since its shape is often close to the skeleton. But, it may not be accurate for filled shapes where topological attributes are important. Further, the computational cost is very high. In (152), authors proposed a novel approach to similarity assessment of graphical symbols based on Kullback-Leibler divergence, where symbols are represented as 2D kernel densities. Based on the reported results, it offers high accuracy compared with the state-of-the-art. Unfortunately, both methods $(151 ; 152)$ may not provide an accurate solution for complex and composite symbols.

Very recently, in (55), author developed the bridge between the literature of sparse representation and the visual vocabulary construction. More specifically, they apply the learned dictionary algorithm for learning a visual vocabulary based on local descriptors of symbols. Next, they construct a vector model for every symbol based on its sparse representation in the vocabulary, and adapt the tdf-if approach to the sparse representation. For isolated symbols (even deformed and degraded samples), their method performed better than well-known state-of-the-art shape descriptors such as Zernike moments (121) and R-signature (144). The method, however, may not be directly applied to complex and composite symbols (27) (see Fig. 5). 
For more details about shape and symbol recognition, we refer to the works presented in $(35 ; 56 ; 153)$, where usefulness of the shape descriptors for document analysis and a collection of techniques employed for graphical symbols recognition have been reported. Most methods are particularly suited for isolated line symbols, not for composed symbols connected to a complex environment (6; 35). In (56), shape analysis for symbol representation has been comprehensively addressed. It mainly refers to simple isolated $2 D$ binary shapes. In statistical approaches, global signal-based descriptors (20; 85; $105 ; 121 ; 125 ; 144)$ are usually quite fault tolerant to image distortions, since they tend to filter out small change in details. This is unfortunately inappropriate in case where symbols are composed of other known and significant symbols (need not necessarily be connected) as well as texts (see Fig. 5). More precisely, the major drawbacks are due to deformation, composition with other symbols (which, in (20) leads to unstable centroid detection, and thus errors in the ring projection) and occlusion over the boundary (leading to unstable tangents in shape context (105)). Despite those drawbacks, researchers have been integrating descriptors (154-156) as well as combining several classifiers (157) to increase their performance, partially based on the idea presented in (131) that off-the-shelf methods are primarily designed for applications where line symbols are isolated. As an example, in (154), the authors combined several features (such as compactness, ellipticity, angular features and GFD), which would make graphical symbol recognition efficient. In a similar manner, in (158; 159), the authors reported the use of statistical features grouping to partition line drawings into shapes, those shapes represent meaningful parts of the symbols. The method applies to accurate and consistent detection of regions-of-interest (i.e., conversion of a complete line drawing into a set of isolated shapes). But, to form clusters of symbols, the method suffers from high computational cost.

On the whole, in these statistical approaches, signatures are simple with low computational cost (except for a few methods that are especially designed to advance graphical symbol recognition). However, discrimination power and robustness strongly depend on the selection of optimal set of features for each specific application.

\section{Structural and syntactic approaches to symbol recognition}

Besides statistical approaches, another idea is to decompose the symbols into either vector based primitives such as points, segments, lines and arcs or into meaningful parts such as circles, triangles and rectangles. Such peculiar structure of technical documents, which is in the form of a simple set of primitives, naturally induces the use of both structural and syntactic approaches $(6 ; 61 ; 160 ; 161)$ to recognize graphical symbols, for instance. The choice depends on how well (i.e., robust) the representation can be made as well as on the skills to make algorithm faster (considering real-world projects).

Since both approaches use primitives (as basic building blocks), in what follows, we first discuss about primitive extraction. This is then followed by structural and syntactic approaches for graphical symbol recognition, retrieval and spotting.

\subsection{Primitives Extraction}

Considering a raster data, a symbol is naturally represented by the number of pixels. As a consequence, use of key points using local descriptor can be an issue to be discussed. But, it is still unclear which local descriptors are more appropriate and how their performances depend on the selection of key points or regions, since the use of descriptors varies from one application to another. Comprehensive explanations can be found in (162) for general image understanding problem. Vectorization (39) is another preliminary task for extracting primitives like simple lines $(163 ; 164)$ and arcs (165-167) including geometric primitives such as loops and contours or simple shapes like circles and rectangles. 
Fig. 8. An example showing over segmentation. It illustrates two identical shapes (symbols) in different scales produces different number of segments.
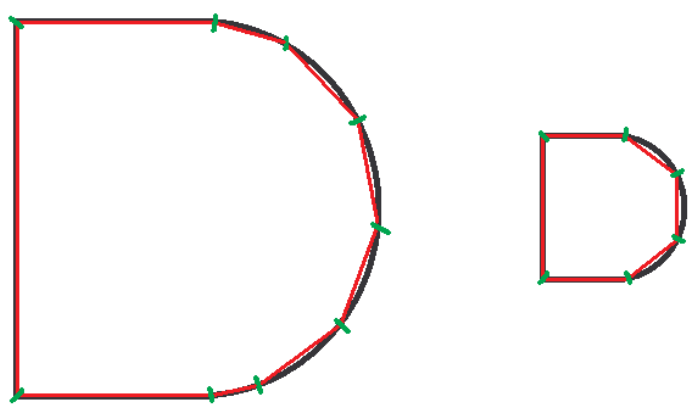

In technical documents, these primitives are considered as key elements since they can represent a complete graphical symbol (168-170). These primitives serve a common basis for both structural and syntactic approaches. It is important to notice that such a set of primitive (meaningful) extraction is not trivial since problems (studied image samples) vary from one application to another.

For line extraction, the Hough transform $(171 ; 172)$ has been widely used. But, applications are reasonably limited in case of degraded scanned document due to its high computational cost. Several approaches are based on the extraction of strings from the skeleton $(173 ; 174)$ having well-known distortion problems but they require a correct localization of junctions (points) (175-178) by matching opposite edges (179). These solutions are limited since the concept is sensitive to the complexity of processed shapes. Motivated by this, the orthogonal zig-zag (OZZ) algorithm (180) and adaptations $(172 ; 181)$ were proposed to make line extraction efficient.

Curves are taken as another subject of research interest. Digital curves have been extracted based on the polygonal approximation in the form of skeleton strings (using the Haar transform, for instance) (182-185). The method leads naturally to a loss of information (or accuracy) with respect to the initial structure but, it offers implementations simplicity for the subsequent algorithms. Other approaches that follow the initial curve are more accurate (186-188). Methods based on dynamic programming are time-consuming but in such a case, code optimizations can help (189-191). Other methods focus on key points that are inserted in the segmentation step (192-195). Most of these approaches require threshold initialization and therefore, they may differ in accordance with the type of the application. As a consequence, developing generic algorithms is not trivial. It is also difficult to combine several different approaches to detect both arcs and segments that are fundamental to ensure accurate symbol recognition. So, it is necessity to avoid over-segmentation (see Fig. 8, for example) since it may generate several small components that will be taken account for matching (196).

Typically these approaches are divided into three categories (197; 198). In arc-fitting methods (166; 178; $192 ; 199)$, the main drawbacks are coming from potential noise and distortions that lead to local errors (i.e., over-segmentation). Other approaches are built from the Hough transform (200; 201). They often produce accurate results in the presence of distortion. But, they require manual threshold initialization, which are sensitive to noise. Further, they are generally expensive in terms of computational cost. Similarly, methods based on stepwise arc extension represent the latest category. They can improve segmentation by studying specific arcs $(165 ; 197 ; 202)$, and remain more stable in most cases.

At this point, vector data require the use of robust extraction operators to ensure analysis and correct understanding of documents $(35 ; 203 ; 204)$. Most of these approaches have been compared in GREC contests: 2001 and 2003 (198; 199; 205; 206), for example. The generic method RANVEC (178; 205) overcomes other methods in most of the problems. One of the drawbacks of this method is that it may omit small parts since it selects points randomly, aiming to reduce the computational time. 


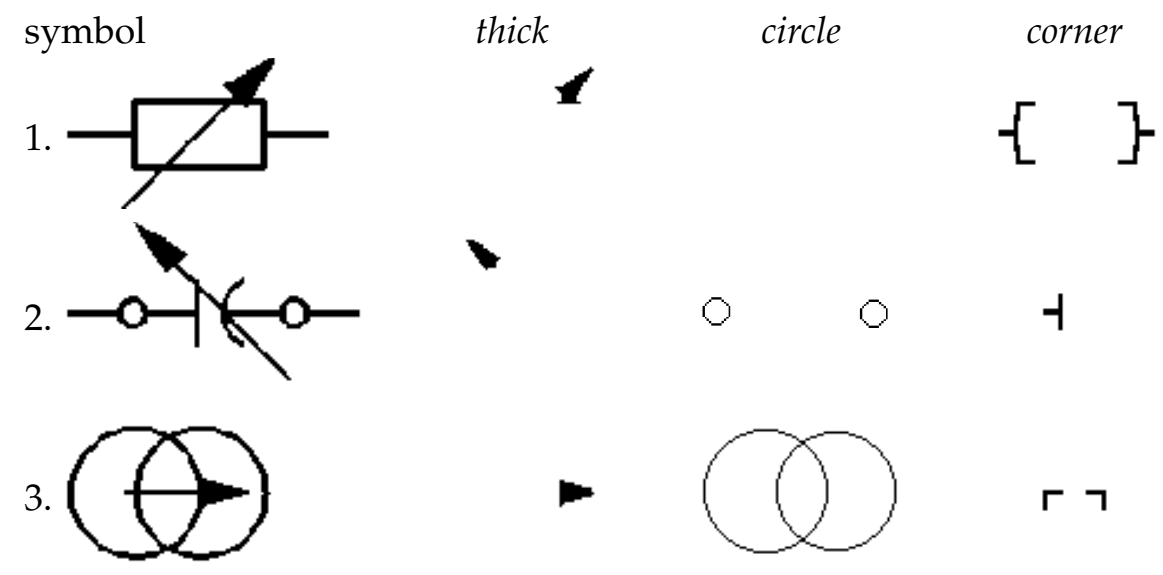

Fig. 9. Three different examples showing visual primitive extraction. Visual primitives are thick (or filled) components, circles and corners (27). The samples are taken from FRESH dataset (71).

Similar primitives such as thick (or filled) components, circles, corners and extremities (i.e., loose end points) have been extracted by using classical image analysis operators $(12 ; 14 ; 26 ; 167)$. Fig. 9 shows a few examples. For thick components, it uses standard skeletonization using chamfer distance and computes the histogram of line thicknesses; and an optimal cut value is computed from the histogram to distinguish between thick zones and thin zones. In (167), the random sample consensus minimization (RANSAC) scheme is used to detect circles and arcs. The performance of the method was demonstrated (as the winner) in the arc segmentation contest on GREC in 2011 (207). Using gradient-direction-based segmentation and direct least-square fitting, a fast and accurate circle detection algorithm, reported in (208) outperform the circular Hough transform (209), the randomized circle Hough transform (210), and the fast circle detection (211) in both processing speed and detection precision. Moreover, very recently, as reported in (212), their method also outperforms RANSAC (167) in arc detection precision and speed. In (213), very recent arc detection contest was reported.

On the whole, considering technical documents (rich in graphics), a common drawback is error-prone raster to vector conversion. For example, primitive types such as arc and corner are not extracted (as expected) as the degradation or noise level increases. However, robust vectorization exists in line drawing images (engineering drawings, for instance) $(177 ; 178 ; 214)$. The use of such low-level primitives varies widely in accordance with the complexity of the symbol.

\subsection{Structural approaches}

Structural approaches are based on symbolic data structures such as strings, trees and graphs. Graphs are most commonly used while strings and trees are always included as special cases. In document analysis, the most recent advancement in graph-based pattern recognition is presented in (215). The formal concept of graphs can be found in (216).

Unlike statistical approaches, structural approaches provide a powerful representation, conveying how parts are connected to each other, while also preserving generality and extensibility (217-220). Graph-based or graph-like representation provides an abstract concept of the studied image. Let us elaborate this with some examples. In (221), authors introduced the notion of interest points by considering corners and junctions. Later, it has been represented by using local descriptors such as SIFT features (222). In (223), for example, local descriptor (Harris-Laplace detector (224)) is used to build a proximity graph for any studied symbol. Fig. 10 shows an example of it. In (225) adjacency relations between the segmented regions have been described. Skeletal graph for shape representation is another example. It uses skeleton points, which are categorized into three families: junction, end 
Fig. 10. An example showing proximity graph representation using interest points using local descriptor.

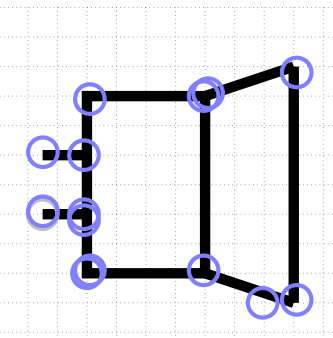

(a) Symbol with interest points

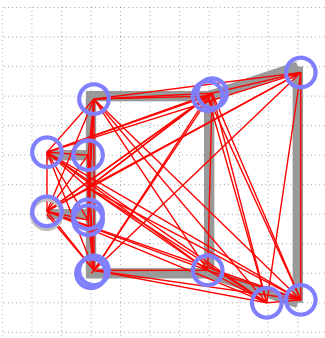

(b) Proximity graph

and branch points. Considering graphical symbol recognition, in (226), a skeletal graph is used to represent the symbol from electrical diagrams. For graph matching, bounded search is used to select the pose of the graph such as rotation, translation and scale for a minimum error transformation. It is entirely based on probabilistic models. In (227), graphs are used for building a model-based scheme for recognizing hand-drawn symbols in schematic diagrams. To construct the graph, as said before, endpoints, junctions and crossings are represented by vertices attributed with the number of neighbours and the angles between incident edges. The edges represent connecting lines in the drawing attributed with the length and curvature of the respective line. Such graph-based representation schemes are not limited; they vary widely such as ARG (228-230), region adjacency graphs (RAG) $(13 ; 231)$, constraint networks (232), deformable templates (15) and proximity graph (223). Similarly, other forms of graph like ARG (specifically designed for symbol representation) (68) provide fundamental parameters related to structural approaches. Fig. 11 shows an example of a line graph. On the whole, they vary from one application to another i.e., a single representation does not fit for all $(233 ; 234)$.

Structural approaches are particularly well suited for recognizing complex symbols (235). Recognizing region-of-interest (graphical symbol, for instance) in a technical document refers to the identification/detection of a part of the graph. The process is known by the name subgraph isomorphism, which is crucial in any real-world context $(236 ; 237)$. This, on the other hand, as said before, leads to a very high computational cost (NP hard problem: polynomial time) (216; 238), which is often the case when complex and composite symbols are taken for evaluation. Further, variability of the size of graphs that can be due to presence of noise and possible distortions leads to computational complexity in matching. Besides, their common drawback comes from error-prone raster-to-vector conversion. This makes symbol representation weak but, varies with the application.

In the framework of stroke-based hand-drawn symbol recognition, prominent studies have been presented in $(239 ; 240)$. The first study is related to template-based matching and another one uses ARGs where the vertices represent geometric primitives like lines and arcs (based on their shapes) and the edges represent the geometric relationships between them. For matching, it is primarily based on graph matching or graph isomorphism detection presented in (241). The work is conceptually similar to $(242)$, which was extended from previous works reported in $(243 ; 244)$. These approaches perform well as long as vertices are well separated since they are taken from on-line strokes. On the whole, this presents an idea of how vectorization difficulties are avoided.

In $(65 ; 245)$, their interest is to map an inaccurate isomorphic structures to address noise artefacts and distorted data by incorporating cost functions for deletion, insertion and node/edge modification. We know that the methods are still sensitive to noise and are suffering from heavy time computational cost even after the integration of the statistical assumptions by using error-tolerant features when searching subgraphs $(13 ; 246)$, for instance. For the same problem, various heuristics are still employed but, they do not guarantee to show significant difference. They however, aim to have a closer to a so-called optimal solution as long as the problem is defined with some constraints. 
Considering the time complexity issue, several works are more focused on computing symbol signatures by taking regions-of-interest (ROIs) in the document image (247-249). These methods aim to provide faster matching in comparison to general graph matching. The performance of such methods is based on how accurate the ROIs are extracted. This means that the methods fail when ROIs do not carry symbols. Further, in (83), author aimed to address how efficiently computational cost can be reduced through the use of graph-based structural pattern recognition approaches. The work has been developed to represent an image by a graph by using the state-of-the-art methods but, a new technique named fuzzy multilevel graph embedding (FMGE) is used to transform that graph to numeric feature vectors (250). As a consequence, it empowers the structural pattern recognition approaches by utilizing statistical pattern recognition tools. Such a graph is used to perform symbol (line drawing) recognition and spotting. While transforming the graphs to numeric feature vectors, there may a loss of information. As a consequence, the FMGE method can be compared with inexact methods, but it may be less accurate than exact methods.

In (251), authors proposed a symbol spotting technique by using graph serialization to reduce the usual computational complexity of graph matching. As said before, graphs are used to represent the documents and a (sub)graph matching technique is used to detect the symbols in them. Serialization of graphs is performed by computing acyclic graph paths between each pair of connected nodes. Graph paths are one-dimensional structures of graphs which are less expensive in terms of computation. At the same time they enable robust localization even in the presence of noise and distortion. For large graph databases, they propose a graph factorization approach to index, which is intended to create a unified indexed structure. Once graph paths are extracted, the entire database of graphical documents is indexed in hash tables by locality sensitive hashing (LSH) of shape descriptors of the paths. The hashing data structure aims to execute an approximate k-NN search in a sub-linear time.

Other methods are based on the relaxation principle based on constraint propagation for matching nodes. Besides high computational cost, another main drawback is that it does not guarantee robustness for correct local matching. These discrete approaches, where a label is associated with each primitive allow a local focusing on inconsistent matching. Such a principle has been used to carry electrical symbol recognition $(252 ; 253)$. Later, Wilson and Handcock have extended the discrete relaxation by introducing a Bayesian model (254). The probabilistic relaxation assigns each node a probability measure according to the constraints. They are then iteratively updated to maximize a measure of consistency on the whole (255-257). Fuzzy concept has also been in use on the relaxation mechanism for handling uncertain data $(258 ; 259)$. Graph-based method allows invariants and is independent of labels (260).

Very recently, in $(261 ; 262)$, authors introduced an interesting approach where a Galois lattice is used to

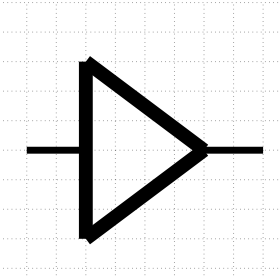

(a) Symbol

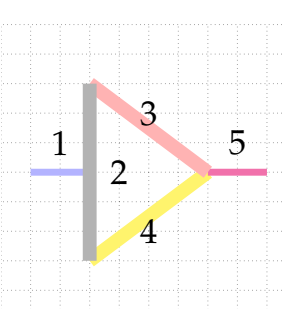

(b) Vectorization (line extraction)

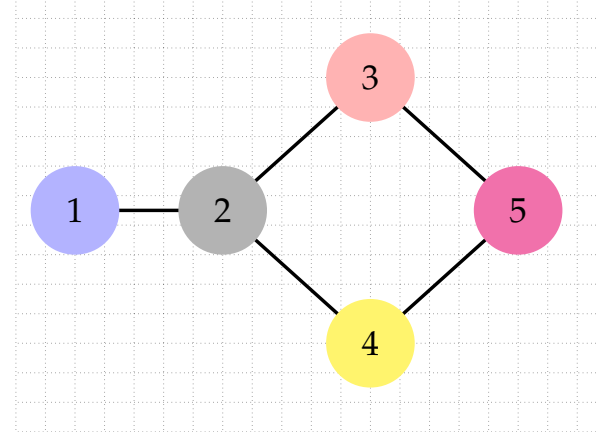

(c) Relational graph

Fig. 11. An example illustrating (a) a symbol, (b) line extraction and (c) its corresponding relational graph. 


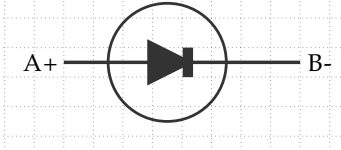

(a) Symbol

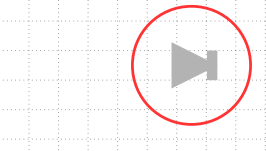

(b) Thick \& Circle

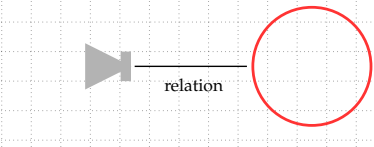

(c) Inside(Thick, Circle)

Fig. 12. An example showing use of spatial relation in the context of symbol recognition. To make it simple, we use spatial predicate (inside, a topological relation) to define the connectivity between the pair of primitives: thick (or filled component) and circle that compose a symbol.

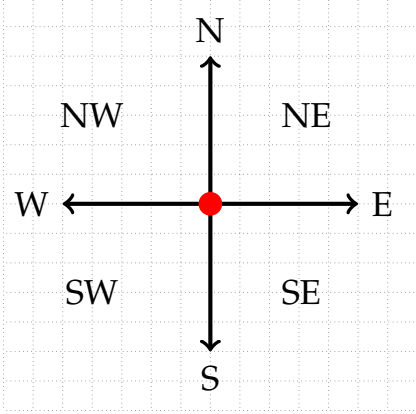

(a) 4-directions

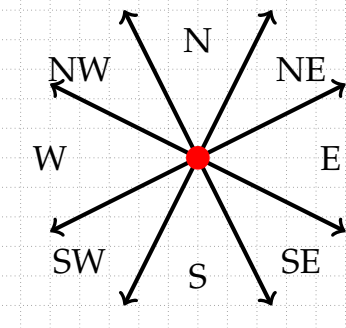

(b) 8-directions

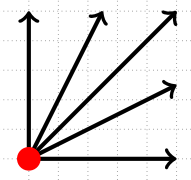

Taken 1st quadra

(c) 16-directions

Fig. 13. Star calculus via bi-centre model using angle-based theory. It shows a cone-based method to compute relations between the studied objects (primitives).

classify structural signatures that are extracted using the Hough transform. These structural signatures are based on a topological graph, where there are only five topological relations computed between the segments (based on connected and disconnected topological configurations). As reported in the paper, the Galois lattice-based classification is robust to noise. However, the performance is inconsistent when symbols are found to be connected with other graphical elements or texts in the whole document image. Later, the advancement has been reported in $(263 ; 264)$.

In (265), authors addressed the problem of symbol spotting in scanned and vectorized line drawings. A set of primitives index the structure of the symbol it is composed of. Such an indexing is used to retrieve similar primitives from the database. Primitives are encoded in terms of attributed strings representing closed regions. Similar strings are clustered in a lookup table so that the set of median strings act as indexing keys. A voting scheme formulates hypothesis in certain locations of the line drawing image where there is a high presence of regions similar to the queried ones, and therefore, a high probability to find the queried graphical symbol. As reported in the paper, the method is found to be robust to noise and distortion, which are introduced by the scanning and raster-to-vector processes. A comprehensive study has been reported in (32).

To handle image recognition, partial recognition of primitives is used to guide the recognition of remaining parts within it (266). In this context, for a complete image recognition, the effect of spatial relations between the primitives on recognition performance has to be determined $(267 ; 268)$. In graph-based pattern representation, connectivity that exists between the nodes must have meaningful information. Spatial reasoning has been considered as a central skill since it is a common and natural way to share spatial information through the use of spatial predicates (269) that ultimately derive relationship between the primitives. To illustrate such a concept, we refer to Fig. 12, where it shows how connectivity between the primitives must be meaningful for recognition. To compute spatial relations, a common angle-based theory based on star-calculus is shown in Fig 13. In document image analysis, precise relations are required to analyze/understand scanned architectural and color map documents (270), recognize models (271), and to define efficient retrieval methods (272-275). 


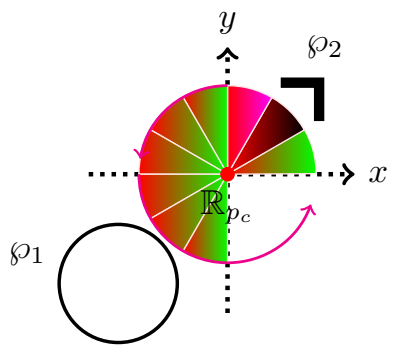

(a) Radial line model
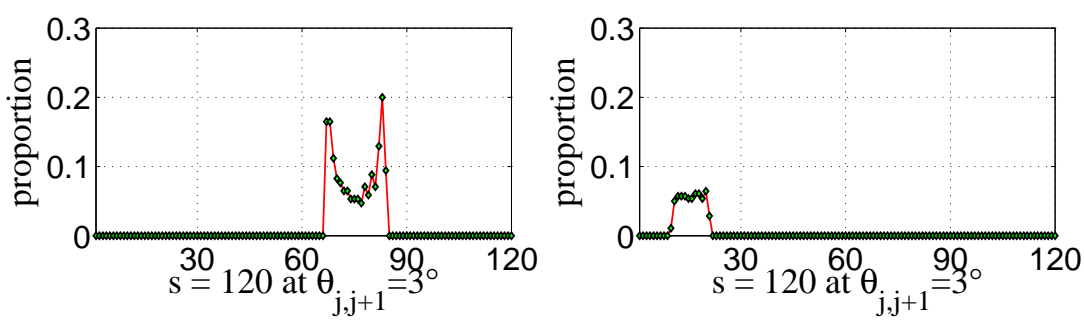

(b) Histograms for both visual primitives: $\wp_{1}$ (left) and $\wp_{2}$ (right).

Fig. 14. Two disconnected pairs of primitives $\wp_{1}$ (circle) and $\wp_{2}$ (corner) and directional relational histograms using the radial line model (RLM) with respect to the unique reference point $\mathbb{R}_{p_{c}}$. RLM is applied for both primitives and the unique reference point is derived using their topological relations.

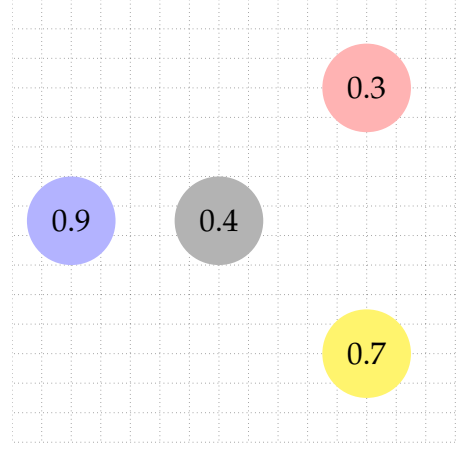

(a) Node features

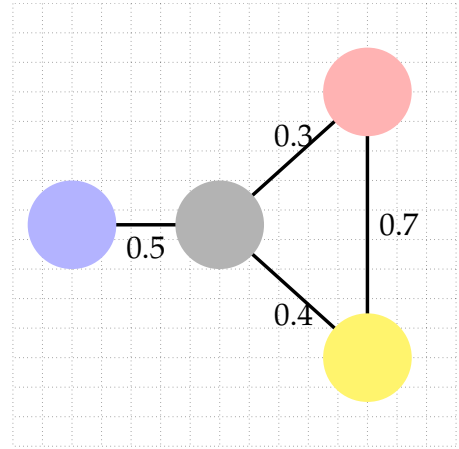

(b) Relational features

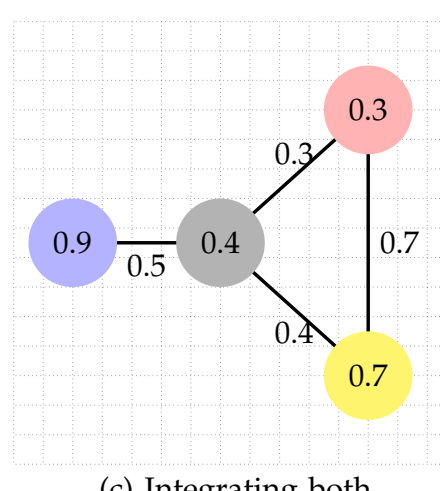

(c) Integrating both

Fig. 15. An example illustrating features using (a) node, (b) relations and (c) integrating both, to make a complete graph.

Similarly, authors have shown the usefulness of relational indexing of vectorial primitives for line drawing images (276). Considering the problem of symbol localization in real documents, composed of individual parts and constrained by spatial relations, as said before, global signal-based descriptors cannot be applied since they are, unfortunately, primarily designed for applications where symbols are isolated. Thus, the problem is related to the segmentation/recognition paradigm (277), for instance, where an accurate segmentation of meaningful parts is expected. In this context (as shown in Fig. 5), we are required to formalize the possible links that exist between them to build a graph-like structure $(28 ; 278 ; 279)$. Considering the real-world problem (see Fig. 5), these methods outperform the state-of-the-art methods used in graphical symbol recognition. Based on this, in (279), the authors tested the use of relational signatures between the possible pairs of labeled vocabulary types such as circles and corners. These relations are computed in terms of histograms as shown in Fig. 14, where the surrounding space is covered by rotating a radial line over a cycle (with a fixed angular step). While rotating, in each sector (made by two consecutive radial lines), histograms are computed for both primitives (i.e., counting the percentage of pixels of the studied primitives lying in it). Both primitives and relational signatures are then used to build an ARG that fully describes the symbol. Since attribute types are fully labeled, corresponding relation alignments are possible between the two graphs. Further, the general NP-hard graph matching problem has been avoided. Very recently, a new concept named Bag-of-Relations (BoRs) for symbol recognition, retrieval and spotting has been introduced (33). The key characteristic of the overall process is to use topological relation information to categorise them in terms of bags and to guide directional relations. The method has been extended to varieties of datasets (symbols) in the domain. Further, usefulness of the method for symbol spotting and for user-friendly symbol retrieval applications has been addressed (33).

In (279), the method is primarily based on the spatio-structural description of primitives. But, it lacks 
information about their shape. Therefore, integrating both features (at nodes and edges) is essential. An example is shown in Fig. 15. Keeping the ARG based symbol description as reported in (279), shape signatures are integrated in two different ways to improve the performance. First, shape signatures are for labelling vertices (278). Second, shape features are applied only to the vocabulary which show significant shape variations, and are grouped via unsupervised clustering (28). In both cases, a major set of well-known state-of-the-shape descriptors are integrated with spatial relations. Overall, this addresses the use of a hybrid approach in symbol recognition since it combines both worlds: structural and statistical, and try to avoid the shortcomings of each of them. Another interesting example integrating shape descriptions with relations to form RAGs is found in (280). The vector-based RAG is based on segmented regions which are labeled as vertices and geometric properties of adjacency relations are used to label edges. However, the approach is limited once segmented regions change with image transformations. Besides, it uses few model classes to localize symbols in technical documents.

\subsection{Syntactic approaches}

Syntactic descriptions of theories and models are at least as powerful for philosophical analysis as descriptions in semantic approaches. Syntactic descriptions are at least as language independent as semantic descriptions. Non-standard models do not pose a severe problem for syntactic descriptions. Syntactic descriptions can be as simple as semantic descriptions, and as close to actual scientific theories (281). Considering graphical symbol recognition, the syntactic approaches are based on formal notions of composition associated with a grammar. The two-dimensional description of the symbols induces the use of graph grammars $(61 ; 282)$. The symbols are recognized by checking whether the representation can be generated by grammar. These methods are powerful tools but they required few noisy data and are especially useful in applications symbols where the shape of the symbols can be accurately described by a rule-set (grammar). Rosenfeld provided a detailed explanation about array, tree and graph grammars (283). In (284), graph grammars can be extended to interpret complex diagrams. Graph grammars were first derived to diagram recognition and limited to rather simple analysis of local symbol configurations. In this paper, the build-weed-incorporate (BWI) programming style for graph grammars was introduced. This shows an idea about interaction among physically distant symbols, which is semantically important. A quick survey about graph grammars is found in (285).

Computer aided design (CAD) projects are based on a human design with well-established rules of composition for a descriptive semantics. The quotation of industrial designs, for example, follows specific rules and therefore can be completely described by a two-dimensional grammar $(59 ; 286)$ or by plex grammars (287). In (59), the authors represent syntactic analysis of the dimensions by representing undirected, labeled graphs called webs. Based on the conventions of web grammar, a set of rules is established. These rules specify how all the possible dimension-sets can be generated and detected. Plex grammars are quite powerful and general in nature since they combine the power of string-, tree-, and web-grammars as sub-cases. The only possible relation between primitives in a string produced by a grammar is the concatenation of relations. A more effective approach is to incorporate relations into string representations which are more general than concatenation. However, the schema is very restricted if higher dimensional patterns are to be described (288).

Using grammar, diagram interpretation and full syntactic analysis of engineering drawings have attracted the interest of the researchers $(289 ; 290)$. In (289), attributed programmed graph grammars were introduced in this paper and their application to the interpretation of schematic diagrams was proposed. In this work, the grammar is used as a generative tool to extract a description of an input diagram. Author studied two classes of diagrams: circuit diagrams and flowcharts. In (290), the authors proposed a methodology to interpret engineering drawings, which is based on the combination 


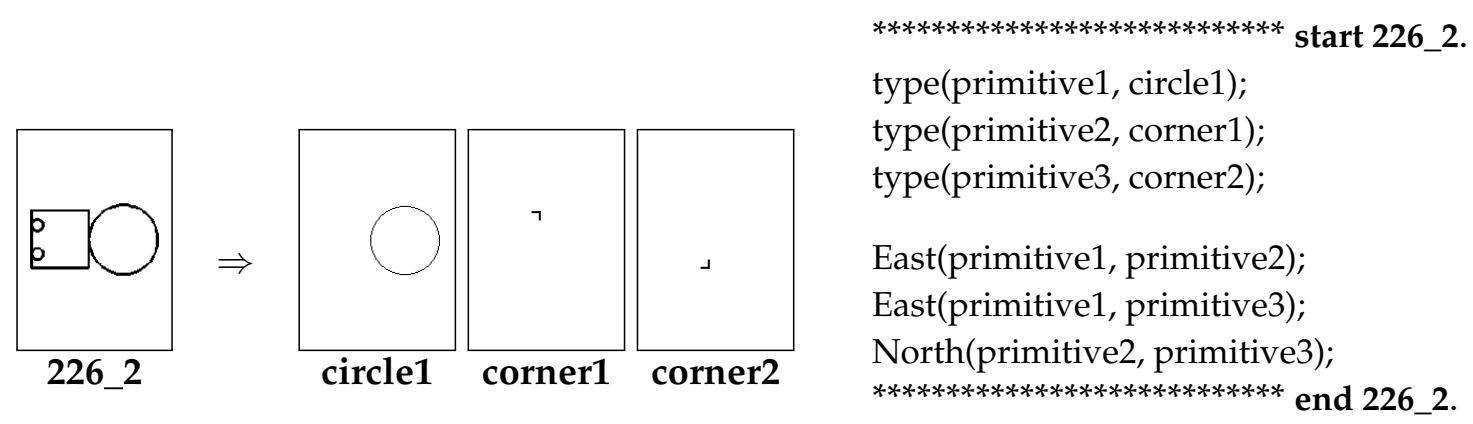

(a) A symbol and its visual primitives

(b) First order logic description

Fig. 16. An example of a formal description (64). It uses spatial relations to define the how the primitives are connected.

of schemata describing prototypical drawing. They constructed it with a library of low-level image analysis routines and a set of explicit control rules by using parser.

Texture recognition in architectural plans can be taken as another support for building graph grammars (291). In their work, a graph grammar is constructed to model textured symbols by inferring a graph grammar from a structured texture, and the subsequent parser to decide whether a symbol is accepted by the grammar. They defined a textured symbol as a symbol consisting of repetitive structured patterns. The grammar is based on a region adjacency graph representation of the vectorized document and the productions are based on the neighboring relations of the patterns forming the textured symbol. Later, in (292), the work has been extended to compute a representative among a class of shapes (i.e., a clustering procedure) in the graphics recognition domain. Few approaches were integrated in the process of vectorization (214) to allow a gradual simplification of data by extraction of the basic shapes to be used in the second step. Other approaches combine structural and syntactic appearance to improve the recognition of specific symbols in documents rich in graphics (217).

Yajie et al. 2007 proposed a novel syntactic symbol recognition approach to the vector based symbol recognition problem (293). In contrast to existing syntactic approaches, which usually describe the geometric relations among primitives, their method formulates a new model to describe the geometric information of a primitive with respect to the whole symbol object based on mathematical analysis. The mathematical model is theoretically rotation and scale invariant and experiments show its accuracy for vector based symbol recognition.

Very recently, in (64), the authors reported the use of formal learning techniques to characterize symbols. The method uses an inductive learning programming (ILP) tool to automatically learn non-trivial descriptions of symbols, based on a formal description. The overall goal of the approach is to express graphical symbols by a number of primitives that may be of any complexity (i.e. not necessarily just lines or points) and connecting relationships that can be deduced from straightforward state-of-the art image treatment and analysis tools. An example of a formal description is shown in Fig. 16. This representation is then used as an input to an ILP solver, in order to deduce non obvious characteristics that may lead to a more semantic related recognition process. For evaluation, it uses a subset of composite symbols from FRESH dataset, as reported earlier.

On the whole, based on the literature, we have found that the syntactic approaches are limited and are varied from one application to another. One of the primary reasons is that it is not trivial to transform statistical signatures into spatial predicates such as disconnected, inside, left and right. The transformation is also inaccurate and thus the image description does not carry complete information. 


\section{Summary}

In this article, we have revised a very challenging research topic: graphical symbol recognition - a sub-field of a larger research domain i.e., pattern recognition - which has been considered as a key problem toward content understanding and interpretation of technical document. In general, starting with its definition, we have discussed basic steps used in state-of-the-art methods, major applications, research standpoints based on several dedicated methods for symbol recognition.

In the 60s and 70s, the resource constrained machines did not allow the use of complex recognition techniques (294) and few data were processed. Since then, the development of more powerful computer machines, interactions between disciplines and the introduction of new applications (data mining, creating taxonomy of documents) led the development of several concepts (41). Graphics recognition has had an extremely rich state-of-the-art literature in symbol recognition and localization since the 70 's $(9 ; 34)$. In the literature, state-of-art methods are categorized into three approaches: statistical, structural and syntactic.

Based on our comprehensive review, statistical approaches are best suited to recognize isolated symbols since they are robust to noise and occlusions. As a consequence, deformed and degraded symbols are recognized well. Statistical signatures are simple (1D vector) to compute with low computational cost. However, as said before, discrimination power and robustness strongly depend on the selection of an optimal set of features as well as proper fusion of classifiers. In contrast, structural approaches are particularly well suited for recognizing complex symbols. For example, they are designed to recognize region-of-interest (graphical symbol, for instance) in a technical document. Structural approaches are based on symbolic data structures such as strings, trees and graphs. Graph matching has been found as a prominent tool in the state-of-the-art methods even though it suffers from high computational cost. As a reminder, graph matching cost often increases when complex and composite symbols are taken for studied due to the well known subgraph isomorphism problem. Further, graph size variability (due to presence of noise and possible distortions) is another cause to increase graph matching cost. Unlike statistical approaches, structural approaches provide a powerful representation, conveying how parts are connected to each other, while also preserving generality and extensibility. The latter term 'extensibility' lets the method can be integrated to other methods from other approaches. Besides, error-prone raster-to-vector conversion is another common drawback. This refers to the primitive extraction step, which is application dependent and therefore, one can focus on unique primitives that are useful for discriminating symbols in that particular application. Therefore, graphs vary from one application to another: proximity graph and line graph, for instance. Note that proximity graph uses interest points (using local descriptors) while, line graph uses lines extracted from the whole document. The latter graph is well suited for technical line drawing images. Syntactic approaches describe symbols (or technical documents) by using well mastered grammars (or rule-set). Needless to say, they use similar primitives as in structural approaches. Interestingly, the syntactic description is close to the language used for human understanding (first order logic description as shown in Fig. 16). But, statistical signatures to spatial predicates transformation may not carry precise metrical details. As a consequence, syntactic approaches do not possess in depth details for recognizing complex documents. Hybrid approaches are basically designed to improve the performance of the system since not a single method (either from statistical, structural or from syntactic) provides a satisfactory performance. Hybrid approaches try to integrate best of the two worlds: structural and statistical, for instance. These are often dedicated to the localization of a particular symbol according to specific rules or to target a set of arbitrary symbols. Note that the concept used in hybrid approach is different than concept of integrating descriptors and classifiers. 
The methods or concepts have been tested in accordance with the context i.e., defined problem and the provided dataset. The recognition problem is fairly straightforward. It is a process where two (test and model) symbols are matched to check their similarity. The test symbol is said to be recognized as the model from which the highest similarity score is produced. This process can be extended to retrieval if a test query retrieves similar symbols and short lists them based on the decreasing order of similarity. Other works are positioned in different context, where they address the recognition of graphical elements and the localization of significant or known visual parts of a document image. The latter problem has been considered as a symbol spotting, which is basically guided by user queries. Query can be a isolated test symbol or just a graphical element that signifies the common property of a group of train symbols. To evaluate the proposed methods, common performance measurement tools are recognition rate (accuracy), precision and recall, F-measure, ROC curve, and confusion matrices (295). Computing these evaluation metrics (precision and recall) is not obvious when ground-truths are uncertain and missed (296). Further, retrieval efficiency is considered as a retrieval quality measure in case of datasets where the number of similar symbols (i.e., ground-truth) varies a lot from one query to another (imbalanced but labelled ground-truths) (297). The context often happens in real-world problem and has been recently studied (27).

Even though state-of-the-art methods are not generic in nature, applications in graphical symbol recognition are not limited. For example, arrow detection, which is basically designed for technical documents (16), has recently been considered as an important step in biomedical images to advance the CBIR problem (298). In both cases, they aim to address regions-of-interest. This is an example that attests the fact that graphical symbol recognition problem can attract a large audience.

\section{Acknowledgements}

The authors would like to thank the NIH Fellows Editorial Board for their editorial assistance.

\section{References}

[1] L. A. Fletcher and R. Kasturi. A robust algorithm for text string separation from mixed text/graphics images. IEEE Transactions on Pattern Analysis and Machine Intelligence, 10(6):910-918, 1988.

[2] G. Nagy. Twenty Years of Document Image Analysis in PAMI. IEEE Transactions on Pattern Analysis and Machine Intelligence, 22(1):38-62, January 2000.

[3] Rangachar Kasturi, Lawrence O'Gorman, and Venu Govindaraju. Document image analysis: A primer. Character Recognition, 27(1):3-22, 2002.

[4] K. Tombre. Analysis of Engineering Drawings: State of the Art and Challenges. In Tombre and Chhabra (299), pages 257-264.

[5] K. Tombre. Ten Years of Research in the Analysis of Graphics Documents: Achievements and Open Problems. In Proceedings of 10th Portuguese Conference on Pattern Recognition, Lisbon, Portugal, pages 11-17, March 1998.

[6] J. Lladós, E. Valveny, G. Sánchez, and E. Martí. Symbol Recognition: Current Advances and Perspectives. In D. Blostein and Y.-B. Kwon, editors, Graphics Recognition - Algorithms and Applications, volume 2390 of Lecture Notes in Computer Science, pages 104-127. Springer-Verlag, 2002.

[7] Karl Tombre. Graphics recognition: The last ten years and the next ten years. In Proceedings of 6 th IAPR International Workshop on Graphics Recognition, Hong Kong, pages 422-426, 2005. 
[8] Karl Tombre. Graphics recognition - what else? In Jean-Marc Ogier, Wenyin Liu, and Josep Lladós, editors, Graphics Recognition. Achievements, Challenges, and Evolution, volume 6020 of Lecture Notes in Computer Science, pages 272-277. Springer, 2010.

[9] D. Doermann and K. Tombre. Handbook of Document Image Processing and Recognition. Springer-Verlag New York Incorporated, 2014.

[10] A. Okazaki, T. Kondo, K. Mori, S. Tsunekawa, and E. Kawamoto. An Automatic Circuit Diagram Reader with Loop-Structure-Based Symbol Recognition. IEEE Transactions on Pattern Analysis and Machine Intelligence, 10(3):331-341, 1988.

[11] Guihuan Feng, Christian Viard-Gaudin, and Zhengxing Sun. On-line hand-drawn electric circuit diagram recognition using 2d dynamic programming. Pattern Recognition, 42(12):3215-3223, 2009.

[12] P. M. Devaux, D. B. Lysak, and R. Kasturi. A complete system for the intelligent interpretation of engineering drawings. International Journal on Document Analysis and Recognition, 2(2/3):120-131, December 1999.

[13] J. Lladós, E. Martí, and J. J. Villanueva. Symbol Recognition by Error-Tolerant Subgraph Matching Between Region Adjacency Graphs. IEEE Transactions on Pattern Analysis and Machine Intelligence, 23(10):1137-1143, 2001.

[14] Ph. Dosch, K. Tombre, C. Ah-Soon, and G. Masini. A complete system for analysis of architectural drawings. International Journal on Document Analysis and Recognition, 3(2):102-116, December 2000.

[15] E. Valveny and E. Martí. A model for image generation and symbol recognition through the deformation of lineal shapes. Pattern Recognition Letters, 24(15):2857-2867, 2003.

[16] L. Wendling and S. Tabbone. A New Way to Detect Arrows in Line Drawings. IEEE Transactions on Pattern Analysis and Machine Intelligence, 26(7):935-941, July 2004.

[17] A. Rebelo, G. Capela, and Jaime S. Cardoso. Optical recognition of music symbols: A comparative study. International Journal on Document Analysis and Recognition, 13(1):19-31, 2010.

[18] Hanan Samet and Aya Soffer. MARCO: map retrieval by content. IEEE Transactions on Pattern Analysis and Machine Intelligence, 18(8):783-798, 1996.

[19] B. B. Chaudhuri and Utpal Garain. An approach for recognition and interpretation of mathematical expressions in printed document. Pattern Analysis and Applications, 3(2):120-131, 2000.

[20] Pong Chi Yuen, Guo-Can Feng, and Yuan Yan Tang. Printed chinese character similarity measurement using ring projection and distance transform. International Journal of Pattern Recognition and Artificial Intelligence, 12(2):209-221, 1998.

[21] Laurent Heutte, Ali Nosary, and Thierry Paquet. A multiple agent architecture for handwritten text recognition. Pattern Recognition, 37(4):665-674, 2004.

[22] K. C. Santosh, Cholwich Nattee, and Bart Lamiroy. Relative positioning of stroke-based clustering: a new approach to online handwritten devanagari character recognition. Int. J. Image Graphics, 12(2), 2012.

[23] K. C. Santosh and Laurent Wendling. Character recognition based on non-linear multi-projection profiles measure. Frontiers of Computer Science, pages 1-13, 2015.

[24] A. K. Chhabra. Graphic Symbol Recognition: An Overview. In Proceedings of 2nd International Workshop on Graphics Recognition, Nancy (France), pages 244-252, August 1997.

[25] Ernest Valveny, Salvatore Tabbone, Oriol Ramos Terrades, and Emilie Philippot. Performance characterization of shape descriptors for symbol representation. In Graphics Recognition, Springer, Lecture Notes in Computer Science Series, pages 278-287, 2007.

[26] J. Rendek, G. Masini, Ph. Dosch, and K. Tombre. The Search for Genericity in Graphics Recognition Applications: Design Issues of the Qgar Software System. In Proceedings of the 6th IAPR International Workshop on Document Analysis Systems, Florence, (Italy), volume 3163 of Lecture Notes in Computer Science, pages 366-377, September 2004. 
[27] K. C. Santosh. Graphics recognition using spatial relations and shape analysis. PhD thesis, INRIA Institut National Polytechnique de Lorraine, Université de Lorraine, November 2011.

[28] K. C. Santosh, Bart Lamiroy, and Laurent Wendling. Integrating vocabulary clustering with spatial relations for symbol recognition. International Journal on Document Analysis and Recognition, 17(1):61-78, 2014.

[29] Salvatore Tabbone, Laurent Wendling, and Daniel Zuwala. A hybrid approach to detect graphical symbols in documents. In Simone Marinai and Andreas Dengel, editors, Proceedings of International Workshop on Document Analysis Systems, Lecture Notes in Computer Science, pages 342-353. Springer, 2004.

[30] Rashid Jalal Qureshi, Jean-Yves Ramel, Didier Barret, and Hubert Cardot. Spotting symbols in line drawing images using graph representations. In Wenyin Liu, Josep Lladós, and Jean-Marc Ogier, editors, Proceedings of IAPR International Workshop on Graphics Recognition, volume 5046 of Lecture Notes in Computer Science, pages 91-103. Springer, 2008.

[31] Mathieu Delalandre, Ernest Valveny, Tony Pridmore, and Dimosthenis Karatzas. Generation of synthetic documents for performance evaluation of symbol recognition \& spotting systems. International Journal on Document Analysis and Recognition, 13(3):187-207, 2010.

[32] Marçal Rusiñol and Josep Lladós. Symbol Spotting in Digital Libraries: Focused Retrieval over Graphic-rich Document Collections. Springer-Verlag London, 2010.

[33] K. C. Santosh, Laurent Wendling, and Bart Lamiroy. Bor: Bag-of-relations for symbol retrieval. International Journal of Pattern Recognition and Artificial Intelligence, 28(06):1450017, 2014.

[34] H. Bunke and P. S. P. Wang, editors. Handbook of Character Recognition and Document Image Analysis. World Scientific, 1997.

[35] L. P. Cordella and M. Vento. Symbol recognition in documents: a collection of techniques? International Journal on Document Analysis and Recognition, 3(2):73-88, December 2000.

[36] M. Robinson, L. S. Baum, J. H. Boose, D. B. Shema, and S. C. Chew. Case Study: Boeing Intelligent Graphics for Airplane Operations and Maintenance. In Conference XML'98, Chicago, USA, December 1998.

[37] S. Adam, J. M. Ogier, C. Cariou, R. Mullot, J. Labiche, and J. Gardes. Symbol and character recognition: application to engineering drawings. International Journal on Document Analysis and Recognition, 3(2):89-101, December 2000.

[38] L. Baum, J. Boose, M. Boose, C. Chaplin, and R. Provine. Extracting System-Level Understanding from Wiring Diagram Manuals. In Proceedings of 5th IAPR International Workshop on Graphics Recognition, Barcelona (Spain), pages 132-138, July 2003.

[39] David S. Doermann. An Introduction to Vectorization and Segmentation. In Tombre and Chhabra (299), pages $1-8$.

[40] R. Kasturi, R. Raman, C. Chennubhotla, and L. O'Gorman. Document Image Analysis: An Overview of Techniques for Graphics Recognition. In Pre-proceedings of IAPR Workshop on Syntactic and Structural Pattern Recognition, Murray Hill, NJ (USA), pages 192-230, 1990.

[41] A. K. Jain, R. P. W Duin, and J. Mao. Statistical Pattern Recognition: A Review. IEEE Transactions on Pattern Analysis and Machine Intelligence, 22(1):4-37, January 2000.

[42] S. Loncaric. A Survey of Shape Analysis Techniques. Pattern Recognition, 31(8):983-1001, 1998.

[43] S. Marshall. Review of shape coding techniques. Image and Vision Computing, 7(4):281-294, 1989.

[44] Ø.D. Trier and T. Taxt. Evaluation of Binarization Methods for Document Images. IEEE Transactions on Pattern Analysis and Machine Intelligence, 17(3):312-315, March 1995.

[45] J. Sauvola and M. Pietikäinen. Adaptive document image binarization. Pattern Recognition, 33(2):225-236, 2000.

[46] U. Garain, T. Paquet, and L. Heutte. On foreground-background separation in low quality document images. International Journal on Document Analysis and Recognition, 8(1):47-63, April 2006. 
[47] S. Ablameyko and O. Okun. Text Separation from Graphics Based on Compactness and Area Properties. Machine Graphics \& Vision, 3(3):531-541, 1994.

[48] Huizhu Luo and Rangachar Kasturi. Improved Directional Morphological Operations for Separation of Characters from Maps/Graphics. In Tombre and Chhabra (299), pages 35-47.

[49] L. Wenyin and D. Dori. A Proposed Scheme for Performance Evaluation of Graphics/Text Separation Algorithms. In Tombre and Chhabra (299), pages 359-371.

[50] R. Cao and C. L. Tan. Text/Graphics Separation in Maps. In D. Blostein and Y.-B. Kwon, editors, Graphics Recognition - Algorithms and Applications, volume 2390 of Lecture Notes in Computer Science, pages 167-177. Springer-Verlag, 2002.

[51] R. Cao and C. L. Tan. Separation of Overlapping Text from Graphics. In Proceedings of the 6th International Conference on Document Analysis and Recognition, Seattle, WA (USA), pages 44-48, September 2001.

[52] K. Tombre, S. Tabbone, L. Pélissier, B. Lamiroy, and Ph. Dosch. Text/graphics separation revisited. In D. Lopresti, J. Hu, and R. Kashi, editors, Proceedings of the 5th IAPR International Workshop on Document Analysis Systems, Princeton, NJ (USA), volume 2423 of Lecture Notes in Computer Science, pages 200-211. Springer-Verlag, August 2002.

[53] A. Velázquez and S. Levachkine. Text/Graphics Separation and Recognition in Raster-scanned Color Cartographic Maps. In Proceedings of 5th IAPR International Workshop on Graphics Recognition, Barcelona (Spain), pages 92-103, July 2003.

[54] Salvatore Tabbone, Laurent Wendling, and Karl Tombre. Matching of graphical symbols in line-drawing images using angular signature information. International Journal on Document Analysis and Recognition, 6(2):115-125, 2003.

[55] DO Thanh Ha. Sparse Representation over Learned Dictionary for Document Analysis. PhD thesis, LORIA, Université de Lorraine, France, 2014.

[56] L. P. Cordella and M. Vento. Symbol and Shape Recognition. In Proceedings of 3rd International Workshop on Graphics Recognition, Jaipur (India), pages 179-186, September 1999.

[57] S. Müller and G. Rigoll. Engineering Drawing Database Retrieval Using Statistical Pattern Spotting Techniques. In Proceedings of 3rd International Workshop on Graphics Recognition, Jaipur (India), pages 219-226, September 1999.

[58] V. Claus, H. Ehrig, and G. Rozenberg, editors. Graph-Grammars and Their Applications to Computer Science and Biology. Lecture Notes in Computer Science. Springer-Verlag, 1979.

[59] D. Dori and A. Pnueli. The grammar of dimensions in machine drawings. Computer Vision, Graphics and Image Processing, 42:1-18, 1988.

[60] M. Flasiński. Characteristics of edNLC-Graph Grammar for Syntactic Pattern Recognition. Computer Vision, Graphics and Image Processing, 47:1-21, 1989.

[61] W. H. Tsai and K. S. Fu. Attributed Grammar: A Tool for Combining Syntactic and Statistical Approaches to Pattern Recognition. IEEE Transactions on Systems, Man, and Cybernetics, 10(12):873-885, 1980.

[62] K. C. You and K. S. Fu. Distorted Shape Recognition Using Attributed Grammars and Error-Correcting Techniques. Computer Vision, Graphics and Image Processing, 13:1-16, 1980.

[63] L. P. Cordella, P. Foggia, R. Genna, and M. Vento. Prototyping Structural Descriptions: An Inductive Learning Approach. In A. Amin, D. Dori, P. Pudil, and H. Freeman, editors, Advances in Pattern Recognition (Proceedings of Joint IAPR Workshops SSPR'98 and SPR'98, Sydney, Australia), volume 1451 of Lecture Notes in Computer Science, pages 339-348, August 1998.

[64] K. C. Santosh, Bart Lamiroy, and Jean-Philippe Ropers. Inductive logic programming for symbol recognition. In Proceedings of International Conference on Document Analysis and Recognition, pages 1330-1334. IEEE Computer Society, 2009.

[65] B. T. Messmer and H. Bunke. Efficient error-tolerant subgraph isomorphism detection. In D. Dori and A. Bruckstein, editors, Shape, Structure and Pattern Recognition (Post-proceedings of 
IAPR Workshop on Syntactic and Structural Pattern Recognition, Nahariya, Israel), pages 231-240. World Scientific, 1995.

[66] GREC. International symbol recognition contest at grec2003, 2003.

[67] Mathieu Delalandre, Jean-Yves Ramel, and Nicolas Sidere. A semi-automatic groundtruthing framework for performance evaluation of symbol recognition and spotting systems. volume 7423 of Lecture Notes in Computer Science, pages 163-172. Springer, 2013.

[68] Mathieu Delalandre, Ernest Valveny, and Josep Lladós. Performance evaluation of symbol recognition and spotting systems: An overview. In Koichi Kise and Hiroshi Sako, editors, Proceedings of International Workshop on Document Analysis Systems, pages 497-505. IEEE Computer Society, 2008.

[69] Marçal Rusiñol and Josep Lladós. A performance evaluation protocol for symbol spotting systems in terms of recognition and location indices. International Journal on Document Analysis and Recognition, 12(2):83-96, 2009.

[70] E. Valveny and Ph. Dosch. Symbol recognition contest: a synthesis. In J. Lladós and Y. B. Kwon, editors, Graphics Recognition: Recent Advances and Perspectives - Selected papers from GREC'03, volume 3088 of Lecture Notes in Computer Science, pages 368-385. Springer-Verlag, 2004.

[71] M. Tooley and D. Wyatt. Aircraft electrical and electronic systems: principles, operation and maintenance. Aircraft engineering principles and practice. Butterworth-Heinemann, 2008.

[72] W. Min, Z. Tang, and L. Tang. Recognition of Dimensions in Engineering Drawings Based on Arrowhead-match. In Proceedings of 2nd International Conference on Document Analysis and Recognition, Tsukuba (Japan), pages 373-376, 1993.

[73] J. E. den Hartog and T. K. ten Kate. Finding Arrows in Utility Maps using a Neural Network. In Proceedings of the 12th International Conference on Pattern Recognition, Jerusalem (Israel), volume 2, pages 190-194, 1994.

[74] G. Priestnall, R. E. Marston, and D. G. Elliman. Arrowhead recognition during automated data capture. Pattern Recognition Letters, 17(3):277-286, March 1996.

[75] D. Doermann. The Indexing and Retrieval of Document Images: A Survey. Computer Vision and Image Understanding, 70(3):287-298, 1998.

[76] Yung-Sheng Chen. Automatic identification for a chinese seal image. Pattern Recognition, 29(11):1807 - 1820, 1996.

[77] Marçal Rusinol and Josep Lladós. Logo spotting by a bag-of-words approach for document categorization. In Proceedings of International Conference on Document Analysis and Recognition, pages 111-115, 2009.

[78] Partha Pratim Roy, Umapada Pal, and Josep Lladós. Seal detection and recognition: An approach for document indexing. In Proceedings of International Conference on Document Analysis and Recognition, pages 101-105, 2009.

[79] Partha Pratim Roy, Umapada Pal, and Josep Lladós. Document seal detection using ght and character proximity graphs. Pattern Recognition, 44(6):1282 - 1295, 2011.

[80] Marçal Rusiñol, Vincent Poulain D'Andecy, Dimosthenis Karatzas, and Josep Lladós. Classification of administrative document images by logo identification. In Young-Bin Kwon and Jean-Marc Ogier, editors, Graphics Recognition. New Trends and Challenges, volume 7423 of Lecture Notes in Computer Science, pages 49-58. Springer Berlin Heidelberg, 2013.

[81] K. Ueda, T. Mutoh, and K. Matsuo. Automatic verification system for seal imprints on japanese bankchecks. In Proceedings of the IAPR International Conference on Pattern Recognition, volume 1, pages 629-632 vol.1, 1998.

[82] Guangyu Zhu and D. Doermann. Logo matching for document image retrieval. In Proceedings of International Conference on Document Analysis and Recognition, pages 606-610, July 2009.

[83] Muhammad Muzzamil Luqman. Fuzzy Multilevel Graph Embedding for Recognition, Indexing and Retrieval of Graphic Document Images. PhD thesis, Francois Rabelais University of Tours France and 
Autonoma University of Barcelona Spain, 2012.

[84] Nibal Nayef. Geomatric-Based Symbol Spotting and Retrieval in Technical Line Drawings. PhD thesis, University of Kaiserslautern, Germany, 2012.

[85] D. Zhang and G. Lu. Review of shape representation and description techniques. Pattern Recognition, 37(1):1-19, 2004.

[86] Satosi Watanabe. Pattern Recognition: Human and Mechanical. John Wiley \& Sons, ISBN: 0471808156, New York, 1985.

[87] H. Kauppinen, T. Seppänen, and M. Pietikäinen. An Experimental Comparison of Autoregressive and Fourier-Based Descriptors in 2D Shape Classification. IEEE Transactions on Pattern Analysis and Machine Intelligence, 17(2):201-207, 1995.

[88] Yong Rui, Alfred She, and Thomas S. Huang. A modified fourier descriptor for shape matching in mars. In S. K. Chang, editor, Image Databases and Multimedia Search, volume 8 of Software Engineering and Knowledge Engineering, pages 165-180. World Scientific Publishing House in Singapore, 1998.

[89] C. H. Lin. New Forms of Shape Invariants from Elliptic Fourier Descriptors. Pattern Recognition, 20:535-545, 1987.

[90] E. Persoon and K. Fu. Shape Discrimination using Fourier Descriptors. IEEE Transactions on Systems, Man, and Cybernetics, 7(3):170-179, 1977.

[91] Dengsheng Zhang and Guojun Lu. Study and evaluation of different fourier methods for image retrieval. Image and Vision Computing, 23(1):33-49, 2005.

[92] Akrem El-ghazal, Otman Basir, and Saeid Belkasim. Farthest point distance: A new shape signature for fourier descriptors. Signal Processing: Image Communication, 24(7):572-586, 2009.

[93] T. Taxt, J. B. Olafsdottir, and M. Daehlen. Recognition of Handwritten Symbols. Pattern Recognition, 23:1155-1166, 1990.

[94] M. Maes. Polygonal Shape Recognition Using String-Matching Techniques. Pattern Recognition, 24(5):433-440, 1991.

[95] Emad Attalla and Pepe Siy. Robust shape similarity retrieval based on contour segmentation polygonal multiresolution and elastic matching. Pattern Recognition, 38(12):2229-2241, 2005.

[96] R. Gerdes, R. Otterbach, and R. Kammuler. Fast and Robust Recognition and Localization of 2D Objects. Machine Vision and Applications, 8:365-374, 1995.

[97] D. H. Ballard. Generalizing the Hough Transform to Detect Arbitary Shapes. Pattern Recognition, 13(2):111-122, 1981.

[98] P. Fränti, A. Mednonogov, V. Kyrki, and H. Kälviäinen. Content-based matching of line-drawing images using the Hough transform. International Journal on Document Analysis and Recognition, 3(2):117-124, December 2000.

[99] A. A. Kassim, T. Tan, and K. H. Tan. A Comparative Study of Efficient Generalised Hough Transform Techniques. Image and Vision Computing, 17:737-748, 1999.

[100] C.-P. Chau and W.-C. Siu. Adaptive dual-point hough transform for object recognition. Computer Vision and Image Understanding, 96(1):1-16, October 2004.

[101] Marcello Pelillo, Kaleem Siddiqi, and Steven W. Zucker. Matching hierarchical structures using association graphs. IEEE Transactions on Pattern Analysis and Machine Intelligence, 21(11):1105-1120, 1999.

[102] Farzin Mokhtarian and Sadegh Abbasi. Shape similarity retrieval under affine transforms. Pattern Recognition, 35(1):31-41, 2002.

[103] Cristina Urdiales, Antonio Bandera, and Francisco Sandoval HernÃąndez. Non-parametric planar shape representation based on adaptive curvature functions. Pattern Recognition, pages 43-53, 2002.

[104] T. Bernier and J-A. Landry. A new method for representing and matching shapes of natural objects. Pattern Recognition, 36(8):1711-1723, 2003. 
[105] S. Belongie, J. Malik, and J. Puzicha. Shape matching and object recognition using shape contexts. IEEE Transactions on Pattern Analysis and Machine Intelligence, 24(4):509-522, 2002.

[106] Benjamin B. Kimia, Allen Tannenbaum, and Steven W. Zucker. Shapes, shocks, and deformations i: The components of two-dimensional shape and the reaction-diffusion space. International Journal of Computer Vision, 15(3):189-224, 1995.

[107] S. C. Zhu and A. L. Yuille. FORMS: A Flexible Object Recognition and Modelling System. International Journal of Computer Vision, 20(3):187-212, 1996.

[108] Daniel Sharvit, Jacky Chan, HÃijseyin Tek, and Benjamin B. Kimia. Symmetry-based indexing of image databases. Journal of Visual Communication and Image Representation, 9:366-380, 1998.

[109] Kaleem Siddiqi, Ali Shokoufandeh, Sven J. Dickinson, and Steven W. Zucker. Shock graphs and shape matching. International Journal of Computer Vision, 35(1):13-32, 1999.

[110] T. B. Sebastian, P. N. Klein, and B. B. Kimia. Recognition of Shapes by Editing Their Shock Graphs. IEEE Transactions on Pattern Analysis and Machine Intelligence, 26(5):550-571, May 2004.

[111] Cho-Huak Teh and Roland T. Chin. On image analysis by the methods of moments. IEEE Transactions on Pattern Analysis and Machine Intelligence, 10(4):496-513, 1988.

[112] S. O. Belkasim, M. Shridar, and M. Ahmadi. Pattern Recognition with Moment Invariants: A Comparative Study and New Results. Pattern Recognition, 24:1117-1138, 1991.

[113] Richard J. Prokop and Anthony P. Reeves. A survey of moment-based techniques for unoccluded object representation and recognition. CVGIP: Graph Models Image Processing, 54(5):438-460, 1992.

[114] R. R. Bailey and M. Srinath. Orthogonal Moment Features for Use with Parametric and non Parametric Classifiers. IEEE Transactions on Pattern Analysis and Machine Intelligence, 18(4):389-399, 1996.

[115] R. Bamieh and R. de Figueiredo. A General Moments Invariants/Attributed Graph Method for the Three Dimensional Object Recognition from a Single Image. IEEE Journal of Robotics and Automation, 2:240-242, 1986.

[116] Y. Chen, N. A. Langrana, and A. K. Das. Perfecting Vectorized Mechanical Drawings. Computer Vision and Image Understanding, 63(2):273-286, March 1996.

[117] R. Teague. Image Analysis via the General Theory of Moments. Journal of the Optical Society of America, 70(8):920-930, 1979.

[118] A. Khotanzad and Y. H. Hong. Invariant Image Recognition by Zernike Moments. IEEE Transactions on Pattern Analysis and Machine Intelligence, 12(5):489-497, 1990.

[119] S. X. Liao and M. Pawlak. On the Accuracy of Zernike Moments for Image Analysis. IEEE Transactions on Pattern Analysis and Machine Intelligence, 20(12):1358-1364, 1998.

[120] M. K. Hu. Visual pattern recognition by moment invariants. IRE Trans. on Information Theory, 8:179-187, 1962.

[121] Whoi-Yul Kim and Yong-Sung Kim. A region-based shape descriptor using zernike moments. Signal Processing: Image Communication, 16(1-2):95 - 102, 2000.

[122] N. K. Kamila, S. Mahapatra, and S. Nanda. Invariance image analysis using modified zernike moments. Pattern Recognition Letters, 26(6):747-753, 2005.

[123] C. Kan and M.D. Srinath. Invariant Character Recognition with Zernike and Orthogonal Fourier-Mellin Moments. Pattern Recognition, 35:143-154, 2002.

[124] C. Chong, P. Raveendran, and R. Mukudan. A comparative analysis of algorithms for fast computation of zernike moments. Pattern Recognition, 36:731-742, 2003.

[125] Dengsheng Zhang and Guojun Lu. Shape-based image retrieval using generic fourier descriptor. Signal Processing: Image Communication, 17(10):825-848, 2002.

[126] B. C. Lin and J. Shen. Fast Computation of Moment Invariants. Pattern Recognition, 24:807-813, 1991.

[127] J. Kittler, M. Hatef, R. Duin, and J. Matas. On combining classifiers. IEEE Transactions on Pattern Analysis and Machine Intelligence, 20(3):226-239, 1998. 
[128] Mineichi Kudo and Jack Sklansky. Comparison of algorithms that select features for pattern classifiers. Pattern Recognition, 33(1):25-41, 2000.

[129] D. Ruta and B. Gabrys. An overview of classifier fusion methods. Computing and Info. Sys., 7(1):1-10, 2000.

[130] R.O. Duda, P.E. Hart, and D.G. Stork. Pattern Classification $-2^{\text {nd }}$ edition. Wiley Interscience Publications, New York, 2001.

[131] K. Tombre, C. Ah-Soon, Ph. Dosch, A. Habed, and G. Masini. Stable, robust and off-the-shelf methods for graphics recognition. In Proceedings of the 14th International Conference on Pattern Recognition, Brisbane (Australia), pages 406-408, August 1998.

[132] Luciano da Fontoura Costa and Roberto M. Cesar Junior. Shape Analysis and Classification: Theory and Practice. CRC Press - Book series on Image Processing, 2001.

[133] R. M. Haralick. Performance Evaluation of Document Image Algorithms. In A. K. Chhabra and D. Dori, editors, Graphics Recognition-Recent Advances, volume 1941 of Lecture Notes in Computer Science, pages 315-323. Springer-Verlag, 2000.

[134] A. Rosenfeld. Image Analysis: Problems, Progress and Prospects. Pattern Recognition, 17(1):3-12, 1984.

[135] A. Rosenfeld and A. C. Kak. Digital Picture Processing, volume 2.8. Academic Press, New York, 1982.

[136] S. K. Pal and A. Rosenfeld. A Fuzzy Medial Axis Transformation Based on Fuzzy Disks. Pattern Recognition Letters, 12(10):585-590, 1991.

[137] W.-Y. Kim and Y.-S. Kim. A new region-based shape descriptor. In in MPEG meeting, TR 15-01, Pisa (Italy), December 1999.

[138] D. Zuwala and S. Tabbone. A Method for Symbol Spotting in Graphical Documents. In H. Bunke and A. L. Spitz, editors, Document Analysis Systems VII: Proceedings of 7th International Workshop on Document Analysis Systems, Nelson (New Zealand), volume 3872 of Lecture Notes in Computer Science, pages 518-528, February 2006.

[139] N. Kita. Object Location Based on Concentric Circular Description. volume 1, pages 637-641, 1992.

[140] S. R. Deans. Applications of the Radon Transform. Wiley Interscience Publications, New York, 1983.

[141] Alexander Kadyrov and Maria Petrou. The trace transform and its applications. IEEE Transactions on Pattern Analysis and Machine Intelligence, 23(8):811-828, 2001.

[142] S. Tabbone, L. Wendling, and K. Tombre. Matching of Graphical Symbols in Line-Drawing Images Using Angular Signature Information. International Journal on Document Analysis and Recognition, 6(2):115-125, 2003.

[143] S. Tabbone and L. Wendling. Binary shape normalization using the Radon transform. In Proceedings of 11th International Conference on Discrete Geometry for Computer Imagery, Naples (Italy), volume 2886 of Lecture Notes in Computer Science, pages 184-193, November 2003.

[144] Salvatore Tabbone, Laurent Wendling, and J.-P. Salmon. A new shape descriptor defined on the radon transform. Computer Vision and Image Understanding, 102(1):42-51, 2006.

[145] Salvatore Tabbone, Oriol Ramos Terrades, and Sabine Barrat. Histogram of radon transform. A useful descriptor for shape retrieval. In Proceedings of the IAPR International Conference on Pattern Recognition, pages 1-4, 2008.

[146] K. C. Santosh, Bart Lamiroy, and Laurent Wendling. DTW for matching radon features: A pattern recognition and retrieval method. In Jacques Blanc-Talon, Richard P. Kleihorst, Wilfried Philips, Dan C. Popescu, and Paul Scheunders, editors, Advances Concepts for Intelligent Vision Systems, volume 6915 of Lecture Notes in Computer Science, pages 249-260. Springer, 2011.

[147] K. C. Santosh, Bart Lamiroy, and Laurent Wendling. Dtw-radon-based shape descriptor for pattern recognition. International Journal of Pattern Recognition and Artificial Intelligence, 27(3):1350008, 2013. 
[148] Alicia Fornés, Josep Lladós, Gemma Sánchez, and Dimosthenis Karatzas. Rotation invariant hand-drawn symbol recognition based on a dynamic time warping model. International Journal on Document Analysis and Recognition, 13(3):229-241, 2010.

[149] Sergio Escalera, Alicia Fornés, Oriol Pujol, Josep Lladós, and Petia Radeva. Circular blurred shape model for multiclass symbol recognition. IEEE Transactions on Systems, Man, and Cybernetics - Part B: Cybernetics, 41(2):497-506, 2011.

[150] Jon Almazán, Alicia Fornés, and Ernest Valveny. A non-rigid appearance model for shape description and recognition. Pattern Recognition, 45(9):3105-3113, 2012.

[151] S. Yang. Symbol Recognition via Statistical Integration of Pixel-Level Constraint Histograms: A New Descriptor. IEEE Transactions on Pattern Analysis and Machine Intelligence, 27(2):278-281, February 2005.

[152] Wan Zhang, Liu Wenyin, and Kun Zhang. Symbol recognition with kernel density matching. IEEE Transactions on Pattern Analysis and Machine Intelligence, 28(12):2020-2024, 2006.

[153] Oriol Ramos Terrades, Salvatore Tabbone, and Ernest Valveny. A review of shape descriptors for document analysis. In Proceedings of International Conference on Document Analysis and Recognition, pages 227-231, 2007.

[154] J. P. Salmon, L. Wendling, and S. Tabbone. Improving the recognition by integrating the combination of descriptors. International Journal on Document Analysis and Recognition, 9(1):3-12, 2007.

[155] Oriol Ramos Terrades, Ernest Valveny, and Salvatore Tabbone. On the combination of ridgelets descriptors for symbol recognition. In Liu Wenyin, Josep Lladós, and Jean-Marc Ogier, editors, Graphics Recognition. Recent Advances and New Opportunities, volume 5046 of Lecture Notes in Computer Science, pages 40-50. Springer-Verlag, 2008.

[156] Sabine Barrat and Salvatore Tabbone. A bayesian network for combining descriptors: application to symbol recognition. International Journal on Document Analysis and Recognition, 13(1):65-75, 2010.

[157] Oriol Ramos Terrades, Ernest Valveny, and Salvatore Tabbone. Optimal classifier fusion in a non-bayesian probabilistic framework. IEEE Transactions on Pattern Analysis and Machine Intelligence, 31(9):1630-1644, 2009.

[158] Nibal Nayef and Thomas M. Breuel. Statistical grouping for segmenting symbols parts from line drawings, with application to symbol spotting. In Proceedings of International Conference on Document Analysis and Recognition, pages 364-368, 2011.

[159] Nibal Nayef and Thomas M. Breuel. Efficient symbol retrieval by building a symbol index from a collection of line drawings. In Richard Zanibbi and Bertrand Coüasnon, editors, Document Recognition and Retrieval, volume 8658 of SPIE Proceedings. SPIE, 2013.

[160] B. T. Messmer and H. Bunke. Automatic Learning and Recognition of Graphical Symbols in Engineering Drawings. In R. Kasturi and K. Tombre, editors, Graphics Recognition-Methods and Applications, volume 1072 of Lecture Notes in Computer Science, pages 123-134. Springer-Verlag, May 1996.

[161] J.-Y. Ramel, G. Boissier, and H. Emptoz. A Structural Representation Adapted to Handwritten Symbol Recognition. In Proceedings of 3rd International Workshop on Graphics Recognition, Jaipur (India), pages 259-266, September 1999.

[162] Krystian Mikolajczyk and Cordelia Schmid. A performance evaluation of local descriptors. IEEE Transactions on Pattern Analysis and Machine Intelligence, 27(10):1615-1630, 2005.

[163] J. Y. Chiang, S. C. Tue, and Y. C. Leu. A New Algorithm for Line Image Vectorization. Pattern Recognition, 31(10):1541-1549, October 1998.

[164] Y. Zheng, H. Li, and D. Doermann. A Parallel-Line Detection Algorithm Based on HMM Decoding. IEEE Transactions on Pattern Analysis and Machine Intelligence, 27(5):777-792, May 2005.

[165] D. Dori. Vector-based Arc Segmentation in the Machine Drawing Understanding System 
Environment. IEEE Transactions on Pattern Analysis and Machine Intelligence, 17(11):1057-1068, November 1995.

[166] Ph. Dosch, G. Masini, and K. Tombre. Improving Arc Detection in Graphics Recognition. In Proceedings of the 15th International Conference on Pattern Recognition, Barcelona (Spain), volume 2, pages 243-246, September 2000.

[167] Bart Lamiroy and Yassine Guebbas. Robust and precise circular arc detection. In Jean-Marc Ogier, Liu Wenyin, and Josep Lladós, editors, Graphics Recognition. Achievements, Challenges, and Evolution, 8th International Workshop, GREC 2009, La Rochelle, France, July 22-23, 2009. Selected Papers, volume 6020 of Lecture Notes in Computer Science, pages 49-60. Springer-Verlag, 2010.

[168] R. Kasturi, S. Bow, J. Gattiker, J. Shah, W. El-Masri, U. Mokate, and S. Honnenahalli. A System for Recognition and Description of Graphics. In Proceedings of 9th International Conference on Pattern Recognition, Rome (Italy), pages 255-259, 1988.

[169] C. C. Shih and R. Kasturi. Extraction of Graphical Primitives from Images of Paper Based Line Drawings. Machine Vision and Applications, 2:103-113, 1989.

[170] D. Lysak and R. Kasturi. Interpretation of Line Drawings with Multiple Views. volume 1, pages 220-222, 1990.

[171] P. Kultanen, E. Oja, and L. Xu. Randomized Hough Transform (RHT) in Engineering Drawing Vectorization System. In Proceedings of IAPR Workshop on Machine Vision Applications, Tokyo (Japan), pages 173-176, 1990.

[172] D. Dori. Orthogonal Zig-Zag: an Algorithm for Vectorizing Engineering Drawings Compared with Hough Transform. Advances in Engineering Software, 28(1):11-24, 1997.

[173] L. Lam, S.-W. Lee, and C. Y. Suen. Thinning Methodologies - A Comprehensive Survey. IEEE Transactions on Pattern Analysis and Machine Intelligence, 14(9):869-885, September 1992.

[174] G. Sanniti di Baja. Well-Shaped, Stable, and Reversible Skeletons from the (3,4)-Distance Transform. Journal of Visual Communication and Image Representation, 5(1):107-115, 1994.

[175] C. S. Fahn, J. F. Wang, and J. Y. Lee. A Topology-Based Component Extractor for Understanding Electronic Circuit Diagrams. Computer Vision, Graphics and Image Processing, 44:119-138, 1988.

[176] R. Kasturi, S. T. Bow, W. El-Masri, J. Shah, J. R. Gattiker, and U. B. Mokate. A System for Interpretation of Line Drawings. IEEE Transactions on Pattern Analysis and Machine Intelligence, 12(10):978-992, 1990.

[177] R. D. T. Janssen and A. M. Vossepoel. Adaptive Vectorization of Line Drawing Images. Computer Vision and Image Understanding, 65(1):38-56, January 1997.

[178] X. Hilaire and K. Tombre. Robust and Accurate Vectorization of Line Drawings. IEEE Transactions on Pattern Analysis and Machine Intelligence, 28(6):890-904, June 2006.

[179] D. Antoine, S. Collin, and K. Tombre. Analysis of Technical Documents: The REDRAW System. In Pre-proceedings of IAPR Workshop on Syntactic and Structural Pattern Recognition, Murray Hill, NJ (USA), pages 1-20, June 1990.

[180] I. Chai and D. Dori. Orthogonal Zig-Zag: An Efficient Method for Extracting Lines from Engineering Drawings. In C. Arcelli, L. P. Cordella, and G. Sanniti di Baja, editors, Visual Form, pages 127-136. Plenum Press, New York and London, 1992.

[181] D. Dori and W. Liu. Sparse Pixel Vectorization: An Algorithm and Its Performance Evaluation. IEEE Transactions on Pattern Analysis and Machine Intelligence, 21(3):202-215, March 1999.

[182] T. J. Davis. Fast Decomposition of Digital Curves into Polygons Using the Haar Transform. IEEE Transactions on Pattern Analysis and Machine Intelligence, 21(8):786-790, August 1999.

[183] P. L. Rosin. Techniques for Assessing Polygonal Approximation of Curves. IEEE Transactions on Pattern Analysis and Machine Intelligence, 19(6):659-666, June 1997.

[184] P. Y. Yin. A New Method of Polygonal Approximation using Genetic Algorithm. Pattern Recognition Letters, 19:1017-1026, 1998. 
[185] P.Y. Yin. A tabu search approach to polygonal approximation of digital curves. International Journal of Pattern Recognition and Artificial Intelligence, 14(2):243-255, 2000.

[186] U. Ramer. An Iterative Procedure for the Polygonal Approximation of Plane Curves. Computer Graphics and Image Processing, 1:244-256, 1972.

[187] K. Wall and P. Danielsson. A Fast Sequential Method for Polygonal Approximation of Digitized Curves. Computer Vision, Graphics and Image Processing, 28:220-227, 1984.

[188] J. Sklansky and V. Gonzalez. Fast Polygonal Approximation of Digitized Curves. Pattern Recognition, 12:327-331, 1980.

[189] J. C. Perez and E. Vidal. Optimum polygonal approximation of digitized curves. Pattern Recognition Letters, 15(8):743-750, August 1994.

[190] A. Kolesnikov and P. Fränti. Data reduction of large vector graphics. Pattern Recognition, 38:381-394, 2005.

[191] M. Salotti. An efficient algorithm for the optimal polygonal approximation of digitized curves. Pattern Recognition Letters, 22(2):215-221, February 2001.

[192] P. L. Rosin and G. A. West. Segmentation of Edges into Lines and Arcs. Image and Vision Computing, 7(2):109-114, May 1989.

[193] C.-H. Teh and R. T. Chin. On the Detection of Dominant Points on Digital Curves. IEEE Transactions on Pattern Analysis and Machine Intelligence, 11(8):859-872, 1989.

[194] W.-Y. Wu and M.-J. J Wang. Detecting the Dominant Points by the Curvature-based Polygonal Approximation. 55:79-88, 1993.

[195] N. Ansari and K. W. Huang. Non-parametric dominant point detection. Pattern Recognition, 24(9):849-862, 1991.

[196] J.-P. Salmon and Laurent Wendling. ARG based on arcs and segments to improve the symbol recognition by genetic algorithm. In Wenyin Liu, Josep Lladós, and Jean-Marc Ogier, editors, Graphics Recognition. Recent Advances and New Opportunities, volume 5046 of Lecture Notes in Computer Science, pages 80-90. Springer, 2007.

[197] J. Song, M. R. Lyu, and S. Cai. Effective Multiresolution Arc Segmentation: Algorithms and Performance Evaluation. IEEE Transactions on Pattern Analysis and Machine Intelligence, 26(11):1491-1506, November 2004.

[198] Liu Wenyin, Jian Zhai, and Dov Dori. Extended summary of the arc segmentation contest. In Dorothea Blostein and Young-Bin Kwon, editors, Graphics Recognition Algorithms and Applications, volume 2390 of Lecture Notes in Computer Science, pages 343-349. Springer, 2002.

[199] Dave Elliman. Tif2vec, an algorithm for arc segmentation in engineering drawings. In Dorothea Blostein and Young-Bin Kwon, editors, Graphics Recognition Algorithms and Applications, volume 2390 of Lecture Notes in Computer Science, pages 350-358. Springer, 2002.

[200] R. S. Conker. A Dual Plane Variation of the Hough Transform for Detecting Non-concentric Circles of Different Radii. Computer Vision, Graphics and Image Processing, 43:115-132, 1988.

[201] V.F. Leavers. The dynamic generalized hough transform: Its relationship to the probabilistic hough transforms and an application to the concurrent detection of circles and ellipses. CVGIP, 56(3):381-398, 1992.

[202] W. Liu and D. Dori. Incremental arc segmentation algorithm and its evaluation. IEEE Trans. Pattern Analysis and Machine Intelligence, 20(4):424-431, 1998.

[203] K. Tombre and D. Dori. Interpretation of Engineering Drawings. In Bunke and Wang (34), chapter 17, pages 457-484.

[204] K. Tombre. Analysis of Engineering Drawings: State of the Art and Challenges. In Proceedings of 2nd International Workshop on Graphics Recognition, Nancy (France), pages 54-61, August 1997.

[205] X. Hilaire. RANVEC and the Arc Segmentation Contest. In D. Blostein and Y.-B. Kwon, editors, Graphics Recognition - Algorithms and Applications, volume 2390 of Lecture Notes in Computer Science, pages 359-364. Springer-Verlag, 2002. 
[206] Liu Wenyin. Report of the arc segmentation contest. In Josep Lladós and Young-Bin Kwon, editors, Graphics Recognition, Recent Advances and Perspectives, volume 3088 of Lecture Notes in Computer Science, pages 364-367. Springer, 2004.

[207] Hasan S. M. Al-Khaffaf, Abdullah Zawawi Talib, and Mohd Azam Osman. Final report of grec'11 arc segmentation contest: Performance evaluation on multi-resolution scanned documents. In Young-Bin Kwon and Jean-Marc Ogier, editors, Proceedings of IAPR International Workshop on Graphics Recognition, volume 7423 of Lecture Notes in Computer Science, pages 187-197. Springer, 2013.

[208] Jianping $\mathrm{Wu}$, Ke Chen, and Xiaohui Gao. Fast and accurate circle detection using gradient-direction-based segmentation. J. Opt. Soc. Am. A, 30(6):1184-1192, 2013.

[209] R. O. Duda and P. Hart. Use of the Hough Transformation to Detect Lines and Curves in Pictures. Communications of the ACM, 15(1):11-15, 1972.

[210] Lei Xu, Erkki Oja, and Pekka Kultanen. A new curve detection method: Randomized hough transform (rht). Pattern Recognition Letters, 11(5):331 - 338, 1990.

[211] Ali Ajdari Rad, Karim Faez, and Navid Qaragozlou. Fast circle detection using gradient pair vectors. In Changming Sun, Hugues Talbot, Sébastien Ourselin, and Tony Adriaansen, editors, Proceedings of the Seventh International Conference on Digital Image Computing: Techniques and Applications, pages 879-888. CSIRO Publishing, 2003.

[212] Ke Chen and Jianping Wu. One-dimensional voting scheme for circle and arc detection. J. Opt. Soc. Am. A, 31(12):2593-2602, Dec 2014.

[213] Syed Saqib Bukhari, Hasan S. M. Al-Khaffaf, Faisal Shafait, Mohd Azam Osman, Abdullah Zawawi Talib, and Thomas M. Breuel. Final report of grec'13 arc and line segmentation contest. In Bart Lamiroy and Jean-Marc Ogier, editors, Graphics Recognition. Current Trends and Challenges, volume 8746 of Lecture Notes in Computer Science, pages 234-239. Springer, 2014.

[214] J. Song, F. Su, C.-L. Tai, and S. Cai. An Object-Oriented Progressive-Simplification Based Vectorization System for Engineering Drawings: Model, Algorithm, and Performance. IEEE Transactions on Pattern Analysis and Machine Intelligence, 24(8):1048-1060, August 2002.

[215] Horst Bunke and Kaspar Riesen. Recent advances in graph-based pattern recognition with applications in document analysis. Pattern Recognition, 44(5):1057-1067, 2011.

[216] L.R. Foulds. Graph Theory Applications. Universitext (1979). Springer New York, 1992.

[217] J. Lladós and G. Sánchez. Graph matching versus graph parsing in graphics recognition - A combined approach. International Journal of Pattern Recognition and Artificial Intelligence, 18(3):455-473, 2004.

[218] Antonio Robles-Kelly and Edwin R. Hancock. String edit distance, random walks and graph matching. International Journal of Pattern Recognition and Artificial Intelligence, 18(03):315-327, 2004.

[219] H. Bunke and B. T. Messmer. Recent advances in graph matching. International Journal of Pattern Recognition and Artificial Intelligence, 11(01):169-203, 1997.

[220] Pasquale Foggia, Gennaro Percannella, and Mario Vento. Graph matching and learning in pattern recognition in the last 10 years. International Journal of Pattern Recognition and Artificial Intelligence, 28(1):1450001, 2014.

[221] Hans P. Morevec. Towards automatic visual obstacle avoidance. In Proceedings of International Joint Conference on Artificial Intelligence, pages 584-584, 1977.

[222] David G. Lowe. Distinctive image features from scale-invariant keypoints. International Journal of Computer Vision, 60(2):91-110, 2004.

[223] Marçal Rusiñol and Josep Lladós. Word and symbol spotting using spatial organization of local descriptors. In Proceedings of International Workshop on Document Analysis Systems, pages 489-496, 2008.

[224] K. Mikolajczyk and C. Schmid. Scale \& Affine Invariant Interest Point Detectors. International Journal of Computer Vision, 60(1):63-86, 2004. 
[225] Azriel Rosenfeld. Adjacency in digital pictures. Information and Control, 26(1):24-33, 1974.

[226] F. C. A. Groen, A. C. Sanderson, and J. F. Schlag. Symbol Recognition in Electrical Diagrams Using Probabilistic Graph Matching. Pattern Recognition Letters, 3:343-350, 1985.

[227] S.W. Lee, J.H. Kim, and F.C.A. Groen. Translation- rotation- and scale invariant recognition of hand-drawn symbols in schematic diagrams. International Journal of Pattern Recognition and Artificial Intelligence, 4(1):1, 1990.

[228] H. Bunke and B. T. Messmer. Efficient Attributed Graph Matching and its Application to Image Analysis. In C. Braccini, L. De Floriani, and G. Vernazza, editors, Proceedings of 8th International Conference on Image Analysis and Processing, San Remo (Italy), volume 974 of Lecture Notes in Computer Science, pages 45-55, September 1995.

[229] Bo Gun Park, Kyoung Mu Lee, Sang Uk Lee, and Jin Hak Lee. Recognition of partially occluded objects using probabilistic ARG (attributed relational graph)-based matching. Computer Vision and Image Understanding, 90(3):217-241, 2003.

[230] D. Conte, P. Foggia, C. Sansone, and M. Vento. Thirty years of graph matching in pattern recognition. International Journal of Pattern Recognition and Artificial Intelligence, 18(3):265-298, 2004.

[231] J. Lladós, J. López-Krahe, and E. Martí. A System to Understand Hand-Drawn Floor Plans Using Subgraph Isomorphism and Hough Transform. Machine Vision and Applications, 10(3):150-158, 1997.

[232] C. Ah-Soon and K. Tombre. Architectural Symbol Recognition Using a Network of Constraints. Pattern Recognition Letters, 22(2):231-248, February 2001.

[233] Salim Jouili and Salvatore Tabbone. Towards performance evaluation of graph-based representation. In Proceedings of the IAPR Graph-Based Representations in Pattern Recognition, pages 72-81, 2011.

[234] S. Jouili and S. Tabbone. Hypergraph-based image retrieval for graph-based representation. Pattern Recognition, 45(11):4054-4068, 2012.

[235] K. Tombre, S. Tabbone, and Ph. Dosch. Musings on Symbol Recognition. In Proceedings of 6th IAPR International Workshop on Graphics Recognition, Hong Kong, pages 23-34, August 2005.

[236] A. T. Berztiss. A backtrack procedure for isomorphism of directed graphs. Journal of the ACM, 20(3):365-377, 1973.

[237] J. R. Ullmann. An Algorithm for Subgraph Isomorphism. Journal of the ACM, 23(1):31-42, 1976.

[238] J. L. Balcazar, J. Diaz, and J. Gabarro. Structural Complexity II. EATCS Monographs on Theorical Computer Science, Springer-Verlag, 1990.

[239] Levent Burak Kara and Thomas F. Stahovich. An image-based, trainable symbol recognizer for hand-drawn sketches. Computers \& Graphics, 29(4):501-517, 2005.

[240] WeeSan Lee, Levent Burak Kara, and Thomas F. Stahovich. An efficient graph-based recognizer for hand-drawn symbols. Computers \& Graphics, 31(4):554-567, 2007.

[241] Bruno T. Messmer and Horst Bunke. Efficient subgraph isomorphism detection: A decomposition approach. IEEE Trans. Knowl. Data Eng., 12(2):307-323, 2000.

[242] X. Xiaogang, S. Zhengxing, P. Binbin, J. Xiangyu, and L. Wenyin. An online composite graphics recognition approach based on matching of spatial relation graphs. International Journal on Document Analysis and Recognition, 7(1):44-55, September 2004.

[243] L. Wenyin, Wenjie Qian, and Xiangyu Jin. Smart Sketchpad - An On-line Graphics Recognition System. In Proceedings of the 6th International Conference on Document Analysis and Recognition, Seattle, WA (USA), pages 1050-1054, September 2001.

[244] Yin Liu, Liu Wenyin, and Changjun Jiang. A structural approach to recognizing incomplete graphic objects. In Proceedings of the 17th International Conference on Pattern Recognition, Cambridge (UK), pages 371-375, 2004. 
[245] L. G. Shapiro and R. Haralick. Structural Description and Inexact Matching. IEEE Transactions on Pattern Analysis and Machine Intelligence, 3(5):504-519, 1981.

[246] B. T. Messmer and H. Bunke. A New Algorithm for Error-Tolerant Subgraph Isomorphism Detection. IEEE Transactions on Pattern Analysis and Machine Intelligence, 20(5):493-504, May 1998.

[247] Ph. Dosch and J. Lladós. Vectorial Signatures for Symbol Discrimination. In Proceedings of 5th IAPR International Workshop on Graphics Recognition, Barcelona (Spain), pages 159-169, July 2003.

[248] Marçal Rusiñol and Josep Lladós. Symbol Spotting in Technical Drawings Using Vectorial Signatures. In Proceedings of 6th IAPR International Workshop on Graphics Recognition, Hong Kong, pages 35-45, August 2005.

[249] Liu Wenyin, Wan Zhang, and Luo Yan. An interactive example-driven approach to graphics recognition in engineering drawings. International Journal on Document Analysis and Recognition, 9(1):13-29, 2007.

[250] Muhammad Muzzamil Luqman, Jean-Yves Ramel, Josep Lladós, and Thierry Brouard. Fuzzy multilevel graph embedding. Pattern Recognition, 46(2):551-565, 2013.

[251] Anjan Dutta, Josep Lladós, and Umapada Pal. A symbol spotting approach in graphical documents by hashing serialized graphs. Pattern Recognition, 46(3):752-768, 2013.

[252] R. Mohr and T. C. Henderson. Arc and Path Consistency Revisited. Artificial Intelligence, 28:225-233, 1986.

[253] A. H. Habacha. Reconnaissance de symboles techniques et analyse contextuelle de schémas. Ph.d. thesis, Institut National Polytechnique de Lorraine, Vandœuvre-lès-Nancy, June 1993.

[254] R. C. Wilson and E. R. Hancock. Structural Matching by Discrete Relaxation. IEEE Transactions on Pattern Analysis and Machine Intelligence, 19(6):634-648, June 1997.

[255] O. D. Faugeras and M. Berthod. Improving Consistency and Reducing Ambiguity in Stochastic Labeling: An Optimization Approach. IEEE Transactions on Pattern Analysis and Machine Intelligence, 3:412-423, 1981.

[256] W. J. Christmas, J. Kittler, and M. Petrou. Structural Matching in Computer Vision Using Probabilistic Relaxation. IEEE Transactions on Pattern Analysis and Machine Intelligence, 17(8):749-764, August 1995.

[257] A. Kostin, J. Kittler, and W. Christmas. Object recognition by symmetrised graph matching using relaxation labelling with an inhibitory mechanism. Pattern Recognition Letters, 26(3):381-393, February 2005.

[258] S. Mesadini, R. Khrishnapuram, and Y Choi. Graph matching by relaxation of fuzzy assigments. IEEE Transactions on Fuzzy Systems, 9(1):173-182, 2001.

[259] R. Balasubramaniam, R. Krishnapuram, S. Medasani, S.H. Jung, and Member-Y.S. Choi. Content-based image retrieval based on a fuzzy approach. IEEE Transactions on Knowledge and Data Engineering, 16(10):1185-1199, 2004.

[260] R. C. Wilson and E. R. Hancock. Pattern Vectors from Algebraic Graph Theory. IEEE Transactions on Pattern Analysis and Machine Intelligence, 27(7):1112-1124, July 2005.

[261] Mickaël Coustaty, Karell Bertet, Muriel Visani, and Jean-Marc Ogier. A new adaptive structural signature for symbol recognition by using a galois lattice as a classifier. IEEE Transactions on Systems, Man, and Cybernetics - Part B: Cybernetics, 41(4):1136-1148, 2011.

[262] M. Visani, K. Bertet, and J.-M. Ogier. Navigala: An original symbol classifier based on navigation through a galois lattice. International Journal of Pattern Recognition and Artificial Intelligence, 25(04):449-473, 2011.

[263] Amani Boumaiza and Salvatore Tabbone. Symbol recognition using a galois lattice of frequent graphical patterns. In Michael Blumenstein, Umapada Pal, and Seiichi Uchida, editors, IAPR International Workshop on Document Analysis Systems, pages 165-169. IEEE, 2012.

[264] Amani Boumaiza and Salvatore Tabbone. A novel approach for graphics recognition based on galois lattice and bag of words representation. In Proceedings of International Conference on 
Document Analysis and Recognition, pages 829-833, 2011.

[265] Marçal Rusiñol, Josep Lladós, and Gemma Sánchez. Symbol spotting in vectorized technical drawings through a lookup table of region strings. Pattern Analysis and Applications, 13(3):321-331, 2010.

[266] Moshe Bar and Shimon Ullman. Spatial context in recognition. Perception, 25:324-352, 1993.

[267] Irving Biederman. Perceiving real-world scenes. Science, 177(43):77-80, 1972.

[268] C B Cave and S M Kosslyn. The role of parts and spatial relations in object identification. Perception, 22(2):229-248, 1993.

[269] G. Retz-Schmidt. Various Views on Spatial Prepositions. AI Magazine, pages 95-104, 1988.

[270] J. Silva Centeno. Segmentation of Thematic Maps Using Colour and Spatial Attributes. In Proceedings of 2nd International Workshop on Graphics Recognition, Nancy (France), pages 233-239, August 1997.

[271] J. H. Vandenbrande and A. A. G. Requicha. Spatial Reasoning for the Automatic Recognition of Machinable Features in Solid Models. IEEE Transactions on Pattern Analysis and Machine Intelligence, 15(12):1269-1285, December 1993.

[272] T. Gevers and A. W. M. Smeulders. Enigma: An Image Retrieval System. volume 2, pages 697-700, 1992.

[273] S.-H. Lee and F.-J. Hsu. Spatial Reasoning and Similarity Retrieval of Images Using 2D C-string Knowledge Representation. Pattern Recognition, 25(3):305-318, 1992.

[274] G. Heidemann. Combining spatial and colour information for content based image retrieval. Computer Vision and Image Understanding, 94:234-270, 2004.

[275] S. Medasani and R. Krishnapuram. A fuzzy approach to content-based image retrieval. In Proc. of FUZZ-IEEE, pages 1251-1260, 1997.

[276] Marçal Rusiñol, Agnès Borràs, and Josep Lladós. Relational indexing of vectorial primitives for symbol spotting in line-drawing images. Pattern Recognition Letters, 31(3):188-201, 2010.

[277] Sungsoo Yoon, Yillbyung Lee, Gyeonghwan Kim, and Yeongwoo Choi. New paradigm for segmentation and recognition of handwritten numeral string. In Proceedings of International Conference on Document Analysis and Recognition, pages 205-209, 2001.

[278] K. C. Santosh, Bart Lamiroy, and Laurent Wendling. Spatio-structural symbol description with statistical feature add-on. In Young-Bin Kwon and Jean-Marc Ogier, editors, Graphics Recognition. New Trends and Challenges, volume 7423 of Lecture Notes in Computer Science, pages 228-237. Springer, 2011.

[279] K. C. Santosh, Bart Lamiroy, and Laurent Wendling. Symbol recognition using spatial relations. Pattern Recognition Letters, 33(3):331-341, 2012.

[280] Pierre Le Bodic, Hervé Locteau, Sébastien Adam, Pierre Héroux, Yves Lecourtier, and Arnaud Knippel. Symbol detection using region adjacency graphs and integer linear programming. In Proceedings of International Conference on Document Analysis and Recognition, pages 1320-1324, 2009.

[281] Sebastian Lutz. What's right with a syntactic approach to theories and models? Erkenntnis, pages $1-18,2014$.

[282] M. Viswanathan. Analysis of Scanned Documents - a Syntactic Approach. In H. S. Baird, H. Bunke, and K. Yamamoto, editors, Structured Document Image Analysis, pages 115-136. Springer-Verlag, Heidelberg, 1992.

[283] A. Rosenfeld. Array, Tree and Graph Grammars. In H. Bunke and A. Sanfeliu, editors, Syntactic and Structural Pattern Recognition: Theory and Applications, chapter 4, pages 85-115. World Scientific, 1990.

[284] Hoda Fahmy and Dorothea Blostein. A graph grammar programming style for recognition of music notation. Machine Vision and Applications, 6(2-3):83-99, 1993.

[285] H. Fahmy and D. Blostein. A survey of graph grammars: theory and applications. In International Conference on Pattern Recognition, Vol.II. Conference B: Pattern Recognition Methodology and Systems, 
pages 294-298, 1992.

[286] D. Dori. Dimensioning Analysis: Toward Automatic Understanding of Engineering Drawings. Communications of the ACM, 35(10):92-103, October 1992.

[287] S. Collin and D. Colnet. Syntactic Analysis of Technical Drawing Dimensions. International Journal of Pattern Recognition and Artificial Intelligence, 8(5):1131-1148, 1994.

[288] T. Feder. Plex languages. Info. Sciences, 3:225-241, 1971.

[289] Horst Bunke. Attributed programmed graph grammars and their application to schematic diagram interpretation. IEEE Transactions on Pattern Analysis and Machine Intelligence, 4(6):574-582, November 1982.

[290] S. H. Joseph and T. P. Pridmore. Knowledge-Directed Interpretation of Mechanical Engineering Drawings. IEEE Transactions on Pattern Analysis and Machine Intelligence, 14(9):928-940, September 1992.

[291] G. Sánchez and J. Lladós. A Graph Grammar to Recognize Textured Symbols. In Proceedings of the 6th International Conference on Document Analysis and Recognition, Seattle, WA (USA), pages 465-469, September 2001.

[292] G. Sánchez, J. Lladós, and K. Tombre. A mean string algorithm to compute the average among a set of 2D shapes. Pattern Recognition Letters, 23(1):203-213, January 2002.

[293] Yu Yajie, Wan Zhang, and Liu Wenyin. A new syntactic approach to graphic symbol recognition. In Proceedings of International Conference on Document Analysis and Recognition, volume 1, pages 516-520, Sept 2007.

[294] G. Nagy. State of the Art in Pattern Recognition. Proceedings of the IEEE, 56(5):836-862, 1968.

[295] Th. Gevers and A. W. M. Smeulders. Content-based Image Retrieval by Viewpoint-invariant Image Indexing. Image and Vision Computing, 17(7), 1999.

[296] Bart Lamiroy and Tao Sun. Computing precision and recall with missing or uncertain ground truth. In Young-Bin Kwon and Jean-Marc Ogier, editors, Graphics Recognition. New Trends and Challenge, volume 7423 of Lecture Notes in Computer Science, pages 149-162. Springer, 2013.

[297] Mohan S. Kankanhalli, Babu M. Mehtre, and Jian Kang Wu. Cluster-based color matching for image retrieval. Pattern Recognition, 29:701-708, 1995.

[298] K.C. Santosh, Laurent Wendling, Sameer Antani, and George Thoma. Scalable arrow detection in biomedical images. In Proceedings of the IAPR International Conference on Pattern Recognition, pages 1051-4651. IEEE Computer Society, 2014.

[299] K. Tombre and A. K. Chhabra, editors. Graphics Recognition-Algorithms and Systems, volume 1389 of Lecture Notes in Computer Science. Springer-Verlag, April 1998. 